\title{
Lunar CubeSat Impact Trajectory Characteristics as a Function of Its Release Conditions
}

\author{
Young-Joo Song, ${ }^{1}$ Ho Jin, ${ }^{2}$ and Ian Garick-Bethell ${ }^{2,3}$ \\ ${ }^{1}$ Satellite Ground System Development Team, Satellite Operation Division, Korea Aerospace Research Institute, \\ 169-84 Gwahagno, Yuseong-Gu, Daejeon 305-806, Republic of Korea \\ ${ }^{2}$ School of Space Research, Kyung Hee University, 1 Seocheon-dong, Giheung-gu, Yongin-si, Gyeonggi-do 446-701, Republic of Korea \\ ${ }^{3}$ Department of Earth and Planetary Sciences, University of California, Santa Cruz, 1156 High Street, Santa Cruz, CA 95064, USA \\ Correspondence should be addressed to Ho Jin; benho@khu.ac.kr
}

Received 26 October 2014; Revised 25 February 2015; Accepted 1 March 2015

Academic Editor: Gongnan Xie

Copyright (c) 2015 Young-Joo Song et al. This is an open access article distributed under the Creative Commons Attribution License, which permits unrestricted use, distribution, and reproduction in any medium, provided the original work is properly cited.

\begin{abstract}
As a part of early system design activities, trajectory characteristics for a lunar CubeSat impactor mission as a function of its release conditions are analyzed. The goal of this mission is to take measurements of surface magnetic fields to study lunar magnetic anomalies. To deploy the CubeSat impactor, a mother-ship is assumed to have a circular polar orbit with inclination of 90 degrees at a $100 \mathrm{~km}$ altitude at the Moon. Both the in- and out-of-plane direction deploy angles as well as delta- $V$ magnitudes are considered for the CubeSat release conditions. All necessary parameters required at the early design phase are analyzed, including CubeSat flight time to reach the lunar surface, impact velocity, cross ranges distance, and associated impact angles, which are all directly affected by the CubeSat release conditions. Also, relative motions between these two satellites are analyzed for communication and navigation purposes. Although the current analysis is only focused on a lunar impactor mission, the methods described in this work can easily be modified and applied to any future planetary impactor missions with CubeSat-based payloads.
\end{abstract}

\section{Introduction}

Since the first launch of a CubeSat in 2003, more than one hundred CubeSats have been put in orbit [1]. Indeed, CubeSats have been proven to enable extremely low-cost missions in near Earth orbit with greater launch accessibility. Recently, ideas to apply CubeSat technology to deep space exploration concepts have greatly increased [2]. Over the coming decade, it is expected that diverse science returns could be obtained from extremely low-cost solar system exploration missions with improved CubeSats technologies that are beyond those demonstrated to date [3]. Recently, the NASA Innovative Advanced Concepts (NIAC) program selected interplanetary CubeSats for further investigation to enable a new class of missions beyond low Earth orbit [4]. The potential missions initially considered by NIAC are Mineral Mapping of an Asteroid, Solar System Escape Technology Demonstration, Earth-Sun Sub-L1 Space Weather Monitor, Phobos Sample Return, Earth-Moon L2 Radio Quiet Observatory, and Out-of-Ecliptic Missions [3]. Other than these missions, innovative deep space exploration concepts using CubeSats have also been proposed or studied. For example, a CubeSat on an Earth-Mars free-return trajectory could characterize the hazardous radiation environment before human mission to Mars and watch for potential hazardous Near Earth Objects (NEOs) [5]. Another mission proposes to study asteroid regolith mechanics and primary accretion processes [6].

Since 1992, Korea has been continuously operating more than ten Earth-orbiting satellites and is now expanding its interests to planetary missions. The Korean space program has plans to launch a lunar orbiter and lander around 2020 and also has plans to explore Mars, asteroids, and deep space in the future. Therefore, the Korean aeronautical and space science community has performed numerous related mission studies, and the Korea Aerospace Research Institute (KARI) is performing pre-phase work for the lunar mission. Several preliminary design studies have already been conducted, such as an optimal transfer trajectory analysis [7-16], mapping orbit analysis [17], landing trajectory analysis [18, 19], contact 
schedule analysis [20, 21], link budget analysis [22-24], lander/rover system design [25-29], and candidate payload design analysis [30]. Before 2020, the first Korean lunar pathfinder orbiter mission is scheduled for launch around 2017 with international collaboration. This may not only secure critical technologies but also form a solid basis for the next lunar mission around 2020. The Korean lunar science committee is now working to select the main scientific objectives for the lunar orbiter mission, and one of the candidates is to fly a CubeSat impactor to explore lunar magnetic anomalies and associated albedo features, known as swirls [31].

In 1959, the Soviet spacecraft called "Luna-1" carried a magnetometer to the Moon. Data from "Luna-1" concluded that the Moon has no global magnetic field like Earth's. However, from the Apollo 15 and 16 missions, it was discovered that strongly magnetized materials are distributed all over the Moon's crust. The origin of lunar magnetism is one of the oldest problems that is still debated in the field of lunar science [32]. Understanding the origin of swirls may help to understand not only geological processes, but also space weathering effects on the lunar surface. Although previous lunar missions such as the National Aeronautics and Space Administration's (NASA) Lunar Prospector and the Japan Aerospace Exploration Agency's (JAXA) KAGUYA have also measured lunar magnetic fields, these data are not sufficient to completely characterize magnetic anomaly regions, since they were obtained at high altitudes $(>20 \mathrm{~km})$ [32]. After the completion of its nominal mission, a lunar orbiter could be crashed into a target area to take measurements at low altitudes, just as most of the past lunar orbiters have ended their missions. However, such an impact is not only expensive but also has rare launch accessibility, and, mostly, there are enormous mission demands that need to be performed at low altitude.

For this reason, a new idea is to use a CubeSat carrying a magnetometer as a payload and impact at the target region of interest. Actually, the concept of CubeSat impactor to measure lunar magnetic fields near the surface has already been discussed [32]. In [32], two major lunar transfer scenarios are proposed to deliver the CubeSat impactor. The first option is to use the Planetary HitchHiker (PHH) concept, which is a small spacecraft designed to be accommodated as a secondary payload on a variety of launch vehicles. In this concept, the launch vehicle places the $\mathrm{PHH}$ spacecraft into Geostationary Transfer Orbit (GTO) to reduce mission costs and after insertion into GTO, the PHH spacecraft uses onboard propulsion to cruise to the Moon and, finally, release the CubeSat impactor after appropriate orbital conditions are established. Appropriate orbital conditions to deploy the CubeSat impactor will be established by several Lunar Orbit Insertions (LOI), orbit adjustments, and station-keeping burns as conventional lunar mission sequences. The second concept is to board the CubeSat impactor into a geostationary spacecraft as a payload and deploy it after reaching geostationary orbit (GEO). The released impactor will spiral out to the Moon with its own minimized ion propulsion system, and upon entering the Moon's gravitational sphere of influence, the CubeSat will directly impact the target area without entering lunar orbit. However, these two mission scenarios have several challenging aspects to overcome, for example, longer flight times to reach the lunar orbit (which is expected to be more than 100 days), tolerating large amounts of radiation exposure even though the mission starts from GEO, and, most importantly, establishing a shallow impact angle $(<10 \mathrm{deg})$ during the impact phase to meet the science objectives, which is a more critical factor if a mother-ship is not used [32].

Another promising method to achieve this mission objective might be to fly the lunar CubeSat impactor as one of the scientific payloads on the lunar orbiter. We believe that this approach will partly ease the challenging aspects that have been raised in the previously discussed scenarios, especially establishing the very shallow impact angle. Indeed, one of the major expected contributions of using CubeSats in planetary missions is that a large variety of near-surface science experiments could be performed [2]. Most of planetary exploration to date has been achieved through remote sensing from orbiters or surface exploration by landers. However, such methods are expensive and risky, and the science data gathered from the near-surface can be limited [2]. For these reasons, CubeSat payload planetary missions are vigorously suggested not only for the Moon, but also for Mars, Europa, and other deep space exploration missions. For example, NASA is considering launching a CubeSat-based payload on a future Mars exploration mission around 2016 to early 2020s, using excess capacity on the mission's primary spacecraft [33]. Recently, the Jet Propulsion Laboratory (JPL) selected and funded CubeSat concept studies for the NASA mission Europa Clipper. This mission is aimed for launch around 2025 with multiple CubeSats and science objectives for the released CubeSats in the Jovian system would include reconnaissance for future landing sites, gravity fields, magnetic fields, atmospheric and plume science, and radiation measurements to enhance our understanding of Europa [34].

As we have already developed experimental CubeSats, which are Triplet Ionospheric Observatory CubeSat for Ion, Neutral, Electron, MAgnetic fields (TRIO CINEMA), and Scientific CubeSat with Instruments for Global Magnetic field and rAdiation (SIGMA) [31], their heritage can directly be applied to the lunar impactor mission proposed in this study. For the TRIO CINEMA mission, now in Earth orbit (CINEMA 1 was launched in 2012 and CINEMAs 2 and 3 were launched in 2013), three different institutions collaborated to develop the CINEMA series, UC Berkeley for the 1st CINEMA and Kyung Hee University for the 2nd and 3rd CINEMAs. The CINEMA mission uses magnetometers based on Magneto Resistive (MR) sensors developed by Imperial College [35]. The SIMGA CubeSat, funded by the 2013 Korean CubeSat program from KARI, aims to test a magnetometer in low Earth orbit based on a Miniaturized FluxGate (MFG) [31]. The appropriate magnetometer for the proposed CubeSat impactor mission would be selected through these heritage instruments. For the data transfer between the CubeSat impactor and a mother-ship, we have determined that Ultra High Frequency (UHF) band communication is feasible, and a separation on the night side of the Moon would 
be preferred, but not the essential factor, to avoid solar wind perturbations to the magnetic field [36].

The main objective of this paper is to perform a feasibility study to obtain numerous insights into a proposed lunar CubeSat impactor mission, especially analyzing impact trajectory characteristics and their dependence on release conditions from a mother-ship. Furthermore, the authors believe that these preliminary impact trajectory design studies will be helpful for further detailed system definition and design activities. Therefore, all necessary parameters for the early design phase are analyzed: CubeSat flight time before impact at the lunar surface, impact velocity, cross ranges distance which is measured on the lunar surface, and associated impact angles which are all directly affected by the CubeSat impactor release conditions. Based on our results, benefits and drawbacks are discussed for selected impact trajectories. Relative motions between a mother-ship and the CubeSat impactor are also analyzed for communication and navigation system design purposes, and through the analysis, many challenging aspects are identified that have to be resolved at a further detailed mission design stage. Although the current analysis only considers a lunar CubeSat impactor mission, the methods can easily be modified and applied to other similar missions where CubeSats are released from a mother-ship orbiting around another planet and will certainly have broad implications for future planetary missions with CubeSats. In Section 2, system dynamics, such as equations of motion, the closest approach condition derivation, impact angle calculation, and relative motion geometry between a mother-ship and the impactor, are described to simulate a given impactor mission. Section 3 provides detailed numerical implications and presumptions that are made for the current study, and various simulation results with further studies planned are presented through Section 4. In Section 5, conclusions are summarized with discussions of work that is planned to be performed in the near future.

\section{System Dynamics}

2.1. Equations of Motion. Two-body equations of motion of the CubeSat impactor after release from a mother-ship flying in the vicinity of the Moon can be expressed as

$$
\left[\begin{array}{c}
\dot{\mathbf{R}}_{\text {Cube }} \\
\dot{\mathbf{V}}_{\text {Cube }}
\end{array}\right]=\left[\begin{array}{c}
\mathbf{V}_{\text {Cube }} \\
-\frac{\mu \mathbf{R}_{\text {Cube }}}{\mathbf{R}_{\text {Cube }}^{3}}
\end{array}\right]
$$

with initial conditions of

$$
\left[\begin{array}{l}
\mathbf{R}_{\text {Cube }}(0) \\
\mathbf{V}_{\text {Cube }}(0)
\end{array}\right]=\left[\begin{array}{c}
\mathbf{R}_{\mathrm{SC}}\left(t_{r}\right) \\
\mathbf{V}_{\mathrm{SC}}\left(t_{r}\right)+\Delta \mathbf{V}
\end{array}\right],
$$

where $\mu$ is the gravitational constant of the Moon, $t_{r}$ is the time of the CubeSat impactor release, and $\mathbf{R}$ and $\mathbf{V}$ denote position and velocity vectors expressed in the Moon-centered Moon Mean Equator and IAU vector of epoch J2000 (MMME2000) frame. In each vector, subscripts "SC" and "Cube" indicate the mother-ship and the CubeSat impactor, respectively. $\Delta \mathbf{V}$ is the divert delta- $V$ expressed in M-MME2000 frame which is generated during the impactor deployment process. $\Delta \mathbf{V}$ can be expressed as $\Delta \mathbf{V}=\Delta \mathbf{V}_{\mathrm{POD}}+\Delta \mathbf{V}_{\mathrm{TST}}$, where $\Delta \mathbf{V}_{\text {POD }}$ is the delta- $V$ induced from the Poly Picosatellite Orbital Deployer (P-POD) and $\Delta \mathbf{V}_{\mathrm{TST}}$ is the delta- $V$ induced from a thruster mounted on the CubeSat. Indeed, $\Delta \mathbf{V}_{\mathrm{POD}}$ and $\Delta \mathbf{V}_{\text {TST }}$ should be regarded separately; however, only the overall impulsive $\Delta \mathbf{V}$ is considered in the current simulation for a preliminary analysis. The overall $\Delta \mathbf{V}$ can be transformed from a defined delta- $V$ vector expressed in the mother-ship's Local Vertical/Local Horizontal (LVLH) frame, $\Delta \mathbf{V}^{\mathrm{LVLH}}$, as follows:

$$
\Delta \mathbf{V}=\left[\mathbf{Q}_{1}\right] \Delta \mathbf{V}^{\mathrm{LVLH}},
$$

where $\mathbf{Q}_{1}$ is the direction cosine matrix defined as [37]

$$
\begin{aligned}
& \mathbf{Q}_{1} \\
& =\left[\begin{array}{lll}
\left(\frac{\left(-\mathbf{R}_{\mathrm{SC}} \times \mathbf{V}_{\mathrm{SC}}\right) \times\left(-\mathbf{R}_{\mathrm{SC}}\right)}{\left|\left(-\mathbf{R}_{\mathrm{SC}} \times \mathbf{V}_{\mathrm{SC}}\right) \times\left(-\mathbf{R}_{\mathrm{SC}}\right)\right|}\right)_{x_{\mathrm{SC}}} & \left(\frac{-\mathbf{R}_{\mathrm{SC}} \times \mathbf{V}_{\mathrm{SC}}}{\left|-\mathbf{R}_{\mathrm{SC}} \times \mathbf{V}_{\mathrm{SC}}\right|}\right)_{x_{\mathrm{SC}}} & \left(\frac{-\mathbf{R}_{\mathrm{SC}}}{\left|-\mathbf{R}_{\mathrm{SC}}\right|}\right)_{x_{\mathrm{SC}}} \\
\left(\frac{\left(-\mathbf{R}_{\mathrm{SC}} \times \mathbf{V}_{\mathrm{SC}}\right) \times\left(-\mathbf{R}_{\mathrm{SC}}\right)}{\left|\left(-\mathbf{R}_{\mathrm{SC}} \times \mathbf{V}_{\mathrm{SC}}\right) \times\left(-\mathbf{R}_{\mathrm{SC}}\right)\right|}\right)_{y_{\mathrm{SC}}} & \left(\frac{-\mathbf{R}_{\mathrm{SC}} \times \mathbf{V}_{\mathrm{SC}}}{\left|-\mathbf{R}_{\mathrm{SC}} \times \mathbf{V}_{\mathrm{SC}}\right|}\right)_{y_{\mathrm{SC}}} & \left(\frac{-\mathbf{R}_{\mathrm{SC}}}{\left|-\mathbf{R}_{\mathrm{SC}}\right|}\right)_{y_{\mathrm{SC}}} \\
\left(\frac{\left(-\mathbf{R}_{\mathrm{SC}} \times \mathbf{V}_{\mathrm{SC}}\right) \times\left(-\mathbf{R}_{\mathrm{SC}}\right)}{\left|\left(-\mathbf{R}_{\mathrm{SC}} \times \mathbf{V}_{\mathrm{SC}}\right) \times\left(-\mathbf{R}_{\mathrm{SC}}\right)\right|}\right)_{z_{\mathrm{SC}}} & \left(\frac{-\mathbf{R}_{\mathrm{SC}} \times \mathbf{V}_{\mathrm{SC}}}{\left|-\mathbf{R}_{\mathrm{SC}} \times \mathbf{V}_{\mathrm{SC}}\right|}\right)_{z_{\mathrm{SC}}} & \left(\frac{-\mathbf{R}_{\mathrm{SC}}}{\left|-\mathbf{R}_{\mathrm{SC}}\right|}\right)_{z_{\mathrm{SC}}}
\end{array}\right] .
\end{aligned}
$$

In (4), subscripts $x_{\mathrm{SC}}, y_{\mathrm{SC}}$, and $z_{\mathrm{SC}}$ denote the unit vector component of defined $\mathbf{R}_{\mathrm{SC}}$ in M-MME2000 frame. In addition, $\Delta \mathbf{V}^{\mathrm{LVLH}}$ can also be expressed with the unit vectors as follows [38]:

$$
\begin{aligned}
\Delta \mathbf{V}^{\mathrm{LVLH}}= & \Delta V \cos \left(\alpha\left(t_{r}\right)\right) \cos \left(\beta\left(t_{r}\right)\right) \widehat{\mathbf{i}}_{\mathrm{SC}}^{\mathrm{LVLH}} \\
& +\Delta V \sin \left(\alpha\left(t_{r}\right)\right) \cos \left(\beta\left(t_{r}\right)\right) \widehat{\mathbf{j}}_{\mathrm{SC}}^{\mathrm{LVLH}} \\
& +\Delta V \sin \left(\beta\left(t_{r}\right)\right) \widehat{\mathbf{k}}_{\mathrm{SC}}^{\mathrm{LVLH}}
\end{aligned}
$$

where $\Delta V$ is the divert delta- $V$ magnitude and $\hat{\mathbf{i}}_{\mathrm{SC}}^{\mathrm{LVLH}}, \hat{\mathbf{j}}_{\mathrm{SC}}^{\mathrm{LVLH}}$, and $\widehat{\mathbf{k}}_{\mathrm{SC}}^{\mathrm{LVLH}}$ are the defined comoving unit vectors in the transverse, opposite normal, and along inward radial directions all attached to the mother-ship. The unit vectors are defined as follows: $\widehat{\mathbf{k}}_{\mathrm{SC}}^{\mathrm{LVH}}$ is the unit vector which always points from the mother-ship's center of the mass along the radius vector to the Moon's center; $\hat{\mathbf{j}}_{\mathrm{SC}}^{\mathrm{LVH}}$ is the unit vector that lies in the opposite direction of the mother-ship's angular momentum vector, and $\widehat{\mathbf{i}}_{\mathrm{SC}}^{\mathrm{LVLH}}$ is the unit transverse vector perpendicular to both $\widehat{\mathbf{k}}_{\mathrm{SC}}^{\mathrm{LVLH}}$ and $\hat{\mathbf{j}}_{\mathrm{SC}}^{\mathrm{LVLH}}$ that points in the direction of the mothership's velocity vector. In Figure 1, the defined geometry of the CubeSat impactor release conditions from the mother-ship is shown.

At the time of the CubeSat impactor release, $t_{r}$, the defined in-plane direction delta- $V$ deploy angle, $\alpha\left(t_{r}\right)$, is measured from the unit vector, $\hat{\mathbf{i}}_{\mathrm{SC}}^{\mathrm{LVLH}}$, to the projected vector onto the local horizontal plane that is perpendicular to the orbital plane. The out-of-plane delta- $V$ direction deploy angle, $\beta\left(t_{r}\right)$, is measured from the local horizontal plane to the delta- $V$ vector in the vertical direction. Therefore, angles $\alpha\left(t_{r}\right)$ and $\beta\left(t_{r}\right)$ can be regarded as the mother-ship's "yaw" and "pitch" attitude orientation angle at the release moment. 


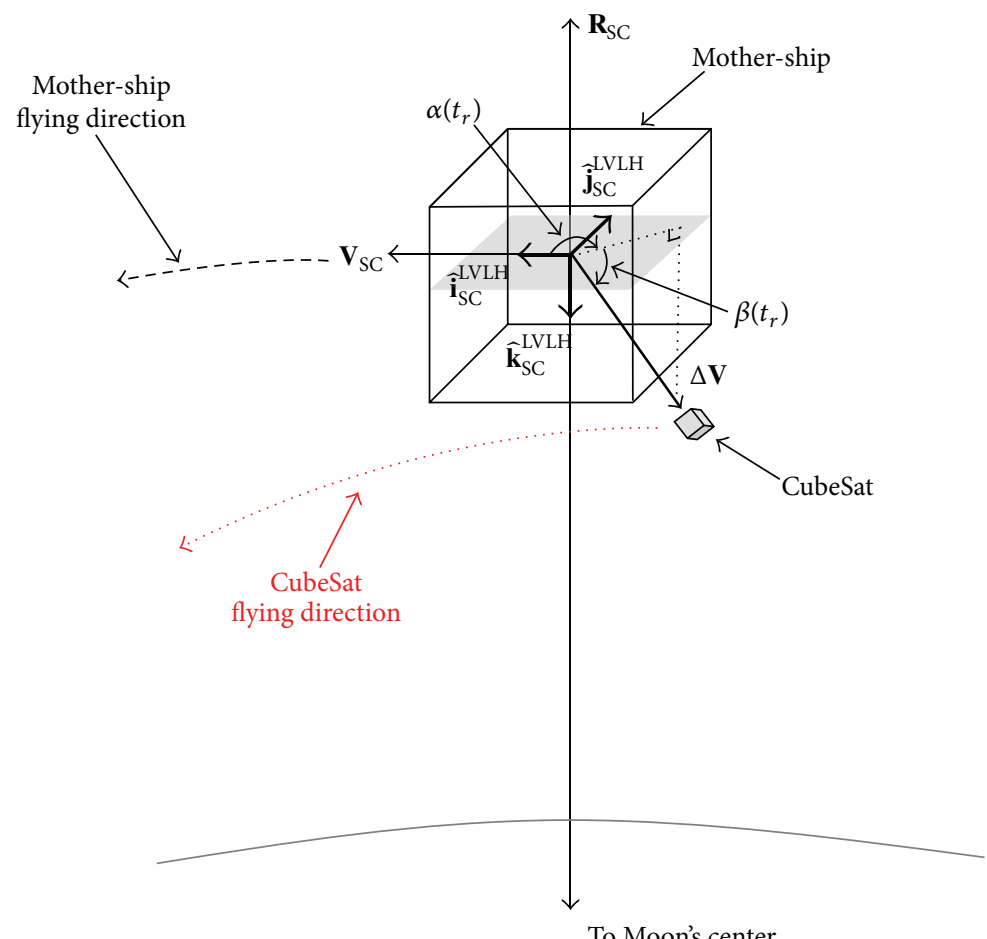

FIGURE 1: Defined geometry of the CubeSat impactor release conditions from the mother-ship (not to scale).

2.2. The Closest Approach Condition. After separation, the closest approach condition between the CubeSat and the lunar surface is computed using the method described in [39], which finds the root of a numerically integrated single nonlinear equation. For numerical integration, the RungeKutta-Fehlberg 7-8th order variable step size integrator is used. During the root-finding process, the objective function, $f_{\text {obj }}$, is given as follows:

$$
f_{\text {obj }}\left(t_{\text {app }}\right)=\left|h_{\text {Cube }}\right| \text {. }
$$

Utilizing methods described in [39], the CubeSat impactor's closest approach time to the lunar surface, $t_{\mathrm{app}}$, and the associated areodetic altitude, $h_{\text {Cube }}$, can be computed by determining whether the given objective function is increasing or decreasing with user specified lower, $t_{\text {app }}^{\text {low }}$, and upper, $t_{\mathrm{app}}^{\mathrm{up}}$, bounds of time search interval and convergence criterion, $\varepsilon_{\text {root }}$. In this study, $h_{\text {Cube }}$ is computed assuming a lunar flatting coefficient, after conversion of the CubeSat's states expressed in the M-MME2000 frame into the Moon Mean Equator and Prime Meridian (M-MMEPM) frame. If $h_{\text {Cube }}$ is found to be greater than $0 \mathrm{~km}$, then the CubeSat will not impact the lunar surface and will fly over with determined $h_{\text {Cube }}$ at $t_{\text {app }}$. If $h_{\text {Cube }}$ is determined to be $0 \mathrm{~km}$, then there will be a lunar surface impact at $t_{\text {app }}$, and, thus, $t_{\text {app }}$ can be regarded as the CubeSat impact time, $t_{\text {imp }}$, or the CubeSat flight time (CFT). In addition, at $t_{\text {imp }}$, the areodetic longitude and latitude of the impact point $\left(\lambda\left(t_{\text {imp }}\right), \phi\left(t_{\text {imp }}\right)\right)$

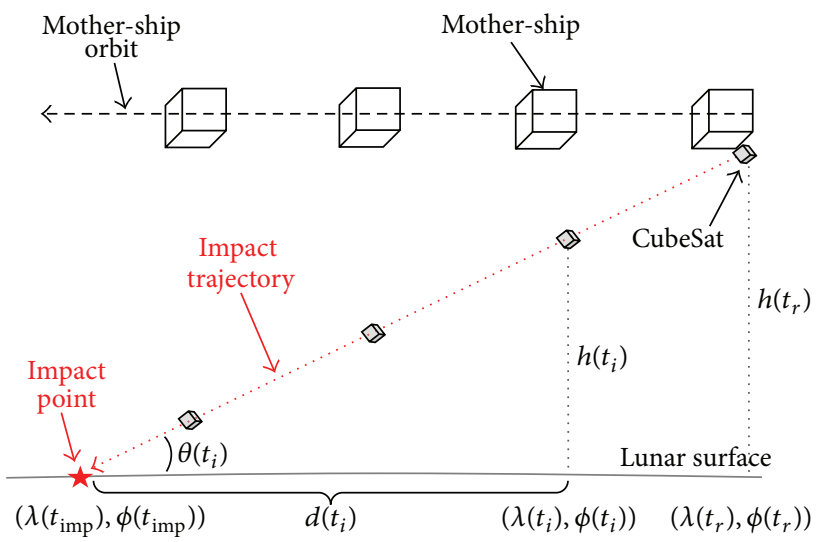

Figure 2: Geometry of the defined CubeSat impact angle (not to scale).

can be easily obtained from the states components expressed in the M-MMEPM frame.

2.3. The CubeSat Impact Angle. The CubeSat impact angle, $\theta\left(t_{i}\right)$, can be approximated as $\theta\left(t_{i}\right)=\tan ^{-1}\left(h\left(t_{i}\right) / d\left(t_{i}\right)\right)$ [32], where $h\left(t_{i}\right)$ is the mother-ship's areodetic altitude at every instant of moment, $t_{i}$, during the impact phase which has ranges of $t_{r} \leq t_{i}<t_{\text {imp. Also, }} d\left(t_{i}\right)$ is the cross range distance between the subground point where the areodetic altitude is measured at $\left(\lambda\left(t_{i}\right), \phi\left(t_{i}\right)\right)$ and the impact point $\left(\lambda\left(t_{\mathrm{imp}}\right), \phi\left(t_{\mathrm{imp}}\right)\right)$. Thus, the cross range distance can be 
regarded as "travel distance" of the CubeSat measured on the lunar surface after separation. In Figure 2, the defined CubeSat impact angle geometry is shown [32]. The $d\left(t_{i}\right)$ between the $\left(\lambda\left(t_{i}\right), \phi\left(t_{i}\right)\right)$ and $\left(\lambda\left(t_{\text {imp }}\right), \phi\left(t_{\text {imp }}\right)\right)$ is computed using the method described in [40] as follows:

$$
\begin{aligned}
& d\left(t_{i}\right) \quad \\
& =r_{\text {pol }}\left(\left[\left(1+f+f^{2}\right) \delta\right]\right. \\
& +r_{\text {Moon }}\left[\left[\left(f+f^{2}\right) \sin \delta-\left(\frac{f^{2}}{2}\right) \delta^{2} \csc \delta\right]\right] \\
& +\xi\left[-\left(\frac{f+f^{2}}{2}\right) \delta-\left(\frac{f+f^{2}}{2}\right)\right. \\
& +r_{\text {Moon }}^{2}\left[-\left(\frac{f^{2}}{2}\right) \sin \delta \cos \delta\right] \\
& +\xi^{2}\left[\left(\frac{f^{2}}{16}\right) \delta+\left(\frac{f^{2}}{16}\right) \sin \delta \cos \delta\right. \\
& \left.\quad-\left(\frac{f^{2}}{2}\right) \delta^{2} \cot \delta-\left(\frac{f^{2}}{8}\right) \sin \delta \cos ^{3} \delta\right] \\
& \left.+r_{\text {Moon }} \xi\left[\left(\frac{f^{2}}{2}\right) \delta^{2} \csc \delta+\left(\frac{f^{2}}{2}\right) \sin \delta \cos ^{2} \delta\right]\right)
\end{aligned}
$$

In (7), $r_{\text {pol }}$ and $r_{\text {Moon }}$ are the Moon's mean polar radius and mean equatorial radius, respectively. Also, $f$ is the Moon's flattening coefficient, $f=1-\left(r_{\text {pol }} / r_{\text {Moon }}\right)$, and $\delta$ and $\xi$ are the defined parameters as follows:

$$
\begin{gathered}
\delta=\tan ^{-1}\left(\left(\left(\sin \varepsilon_{1} \sin \varepsilon_{2}\right)+\left(\cos \varepsilon_{1} \cos \varepsilon_{2}\right) \cos \lambda\right)\right. \\
\cdot\left(\left(\sin \lambda \cos \varepsilon_{2}\right)^{2}\right. \\
+\left[\sin \left(\varphi_{2}-\varphi_{1}\right)\right. \\
\left.\left.\left.+2 \cos \varepsilon_{2} \sin \varepsilon_{1} \sin ^{2}\left(\frac{\lambda}{2}\right)\right]^{2}\right)^{-1 / 2}\right), \\
\xi=1-\left(\frac{\left(\cos \varepsilon_{1} \cos \varepsilon_{2}\right) \sin \lambda}{\sin \delta}\right)^{2},
\end{gathered}
$$

where

$$
\begin{gathered}
\lambda=\lambda\left(t_{\text {imp }}\right)-\lambda\left(t_{i}\right), \\
\varepsilon_{1}=\tan ^{-1}\left(\frac{r_{\text {pol }} \sin \phi\left(t_{i}\right)}{r_{\text {Moon }} \cos \phi\left(t_{i}\right)}\right),
\end{gathered}
$$

$$
\begin{gathered}
\varepsilon_{2}=\tan ^{-1}\left(\frac{r_{\mathrm{pol}} \sin \phi\left(t_{\mathrm{imp}}\right)}{r_{\text {Moon }} \cos \phi\left(t_{\mathrm{imp}}\right)}\right), \\
\varphi_{2}-\varphi_{1}=\left(\phi\left(t_{\mathrm{imp}}\right)-\phi\left(t_{i}\right)\right) \\
+2\left[\sin \left(\phi\left(t_{\mathrm{imp}}\right)-\phi\left(t_{i}\right)\right)\right] \\
\cdot\left[\left(\eta+\eta^{2}+\eta^{3}\right) r_{\text {Moon }}-\left(\eta-\eta^{2}+\eta^{3}\right) r_{\mathrm{pol}}\right] \\
\eta=\left(\frac{r_{\text {Moon }}-r_{\mathrm{pol}}}{r_{\text {Moon }}+r_{\mathrm{pol}}}\right) .
\end{gathered}
$$

2.4. Relative Motion between the Mother-Ship and CubeSat. After the CubeSat impactor is deployed, relative motion between the mother-ship and CubeSat impactor during the impact phase in M-MME2000 frame can be determined as in

$$
\begin{aligned}
& \mathbf{R}_{\mathrm{SC} 2 \mathrm{C}}=\mathbf{R}_{\mathrm{SC}}-\mathbf{R}_{\text {Cube }}, \\
& \mathbf{V}_{\mathrm{SC} 2 \mathrm{C}}=\mathbf{V}_{\mathrm{SC}}-\mathbf{V}_{\text {Cube }}, \\
& \mathbf{R}_{\mathrm{C} 2 \mathrm{SC}}=\mathbf{R}_{\text {Cube }}-\mathbf{R}_{\mathrm{SC}}, \\
& \mathbf{V}_{\mathrm{C} 2 \mathrm{SC}}=\mathbf{V}_{\text {Cube }}-\mathbf{V}_{\mathrm{SC}} .
\end{aligned}
$$

In (10), $\mathbf{R}_{\mathrm{SC} 2 \mathrm{C}}$ and $\mathbf{V}_{\mathrm{SC} 2 \mathrm{C}}$ are the CubeSat impactor's position and velocity vectors seen from a mother-ship, and $\mathbf{R}_{\mathrm{C} 2 \mathrm{SC}}$ and $\mathbf{V}_{\mathrm{C} 2 \mathrm{SC}}$ are the mother-ship's position and velocity vectors seen from the CubeSat impactor during impact phase, respectively. Using (11) (14), $\mathbf{R}_{\mathrm{SC} 2 \mathrm{C}}, \mathbf{V}_{\mathrm{SC} 2 \mathrm{C}}$ and $\mathbf{R}_{\mathrm{C} 2 \mathrm{SC}}, \mathbf{V}_{\mathrm{C} 2 \mathrm{SC}}$ can be expressed with respect to the LVLH frame attached to the mother-ship, $\mathbf{R}_{\mathrm{SC} 2 \mathrm{C}}^{\mathrm{LVLH}}, \mathbf{V}_{\mathrm{SC} 2 \mathrm{C}}^{\mathrm{LVLH}}$, and the LVLH frame attached to the CubeSat impactor, $\mathbf{R}_{\mathrm{C} 2 \mathrm{SC}}^{\mathrm{LVLH}}, \mathbf{V}_{\mathrm{C} 2 \mathrm{SC}}^{\mathrm{LVLH}}$, respectively:

$$
\begin{aligned}
& \mathbf{R}_{\mathrm{SC} 2 \mathrm{C}}^{\mathrm{LVLH}}=\left[\mathbf{Q}_{1}\right]^{T} \mathbf{R}_{\mathrm{SC} 2 \mathrm{C}}, \\
& \mathbf{V}_{\mathrm{SC} 2 \mathrm{C}}^{\mathrm{LVLH}}=\left[\mathbf{Q}_{1}\right]^{T} \mathbf{V}_{\mathrm{SC} 2 \mathrm{C}}, \\
& \mathbf{R}_{\mathrm{C} 2 \mathrm{SC}}^{\mathrm{LVLH}}=\left[\mathbf{Q}_{2}\right]^{T} \mathbf{R}_{\mathrm{C} 2 \mathrm{SC}}, \\
& \mathbf{V}_{\mathrm{C} 2 \mathrm{SC}}^{\mathrm{LVLH}}=\left[\mathbf{Q}_{2}\right]^{T} \mathbf{V}_{\mathrm{C} 2 \mathrm{SC}} .
\end{aligned}
$$

In (13) and (14), $\mathbf{Q}_{2}$ is the direction cosine matrix that transforms a vector from LVLH frame attached to the CubeSat impactor to the CubeSat centered MME2000 frame defined in (15). In (15), subscripts $x_{\text {Cube }}, y_{\text {Cube }}$, and $z_{\text {Cube }}$ denote the unit vector components of $\mathbf{R}_{\text {Cube }}$ defined in the M-MME2000 frame 


$$
\mathbf{Q}_{2}=\left[\begin{array}{lll}
\left(\frac{\left(-\mathbf{R}_{\text {Cube }} \times \mathbf{V}_{\text {Cube }}\right) \times\left(-\mathbf{R}_{\text {Cube }}\right)}{\left|\left(-\mathbf{R}_{\text {Cube }} \times \mathbf{V}_{\text {Cube }}\right) \times\left(-\mathbf{R}_{\text {Cube }}\right)\right|}\right)_{x_{\text {Cube }}} & \left(\frac{-\mathbf{R}_{\text {Cube }} \times \mathbf{V}_{\text {Cube }}}{\left|-\mathbf{R}_{\text {Cube }} \times \mathbf{V}_{\text {Cube }}\right|}\right)_{x_{\text {Cube }}} & \left(\frac{-\mathbf{R}_{\text {Cube }}}{\left|-\mathbf{R}_{\text {Cube }}\right|}\right)_{x_{\text {Cube }}} \\
\left(\frac{\left.-\mathbf{R}_{\text {Cube }} \times \mathbf{V}_{\text {Cube }}\right) \times\left(-\mathbf{R}_{\text {Cube }}\right)}{\left|\left(-\mathbf{R}_{\text {Cube }} \times \mathbf{V}_{\text {Cube }}\right) \times\left(-\mathbf{R}_{\text {Cube }}\right)\right|}\right)_{y_{\text {Cube }}} & \left(\frac{-\mathbf{R}_{\text {Cube }} \times \mathbf{V}_{\text {Cube }}}{\left|-\mathbf{R}_{\text {Cube }} \times \mathbf{V}_{\text {Cube }}\right|}\right)_{y_{\text {Cube }}} & \left(\frac{-\mathbf{R}_{\text {Cube }}}{\left|-\mathbf{R}_{\text {Cube }}\right|}\right)_{y_{\text {Cube }}} \\
\left(\frac{\left(-\mathbf{R}_{\text {Cube }} \times \mathbf{V}_{\text {Cube }}\right) \times\left(-\mathbf{R}_{\text {Cube }}\right)}{\mid\left(-\mathbf{R}_{\text {Cube }} \times \mathbf{V}_{\text {Cube }}\right) \times\left(-\mathbf{R}_{\text {Cube }} \mid\right.}\right)_{z_{\text {Cube }}} & \left(\frac{-\mathbf{R}_{\text {Cube }} \times \mathbf{V}_{\text {Cube }}}{\left|-\mathbf{R}_{\text {Cube }} \times \mathbf{V}_{\text {Cube }}\right|}\right)_{z_{\text {Cube }}} & \left(\frac{-\mathbf{R}_{\text {Cube }}}{\left|-\mathbf{R}_{\text {Cube }}\right|}\right)_{z_{\text {Cube }}}
\end{array}\right] .
$$

Using (11)-(14), the relative location between the mothership and CubeSat impactor during the impact phase, $t=$ $t_{\text {imp }}-t_{r}$, can simply be expressed in terms of in-plane, $\alpha(t)_{\mathrm{SC} 2 \mathrm{C}}$, and out-of-plane direction angle, $\beta(t)_{\mathrm{SC} 2 \mathrm{C}}$, of the CubeSat impactor seen from the mother-ship. Also, in-plane $\alpha(t)_{\mathrm{C} 2 \mathrm{SC}}$ and out-of-plane angles, $\beta(t)_{\mathrm{C} 2 \mathrm{SC}}$, of the mothership are seen from the CubeSat impactor as shown in (16). In Figure 3, geometry of defined relative motions between the mother-ship and CubeSat impactor is shown:

$$
\begin{gathered}
\alpha(t)_{\mathrm{SC} 2 \mathrm{C}}=\tan ^{-1}\left(\frac{\left(\mathbf{R}_{\mathrm{SC} 2 \mathrm{C}}^{\mathrm{LVLH}} /\left|\mathbf{R}_{\mathrm{SC} 2 \mathrm{C}}^{\mathrm{LVLH}}\right|\right)_{j_{\mathrm{SC}}}}{\left(\mathbf{R}_{\mathrm{SC} 2 \mathrm{C}}^{\mathrm{LVLH}} /\left|\mathbf{R}_{\mathrm{SC} 2 \mathrm{C}}^{\mathrm{LVLH}}\right|\right)_{i_{\mathrm{SC}}^{\mathrm{LVH}}}}\right), \\
\beta(t)_{\mathrm{SC} 2 \mathrm{C}}=\sin ^{-1}\left(\left(\frac{\mathbf{R}_{\mathrm{SC} 2 \mathrm{C}}^{\mathrm{LVLH}}}{\left|\mathbf{R}_{\mathrm{SC} 2 \mathrm{C}}^{\mathrm{LVLH}}\right|}\right)_{k_{\mathrm{SC}}^{\mathrm{LVLH}}}\right), \\
\alpha(t)_{\mathrm{C} 2 \mathrm{SC}}=\tan ^{-1}\left(\frac{\left(\mathbf{R}_{\mathrm{C} 2 \mathrm{C} \mathrm{C}}^{\mathrm{LVLH}} /\left|\mathbf{R}_{\mathrm{C} 2 \mathrm{SC}}^{\mathrm{LVLH}}\right|\right)_{j_{\mathrm{Cube}}^{\mathrm{LVH}}}}{\left(\mathbf{R}_{\mathrm{C} 2 \mathrm{~S}}^{\mathrm{LVL}} /\left|\mathbf{R}_{\mathrm{C} 2 \mathrm{SC}}^{\mathrm{LLLH}}\right|\right)_{i_{\mathrm{Cube}}^{\mathrm{LVH}}}^{\mathrm{LVH}}}\right), \\
\beta(t)_{\mathrm{C} 2 \mathrm{SC}}=\sin ^{-1}\left(\left(\frac{\mathbf{R}_{\mathrm{C} 2 \mathrm{SC}}^{\mathrm{LVLH}}}{\left|\mathbf{R}_{\mathrm{C} 2 \mathrm{SC}}^{\mathrm{LVLH}}\right|}\right)_{k_{\mathrm{Cube}}^{\mathrm{LLH}}}\right) .
\end{gathered}
$$

\section{Numerical Implication and Presumptions}

During simulations, several assumptions are made to simplify the given problem as to focus on early design phase analysis. Two-body equations of motion are used to propagate both the mother-ship and the CubeSat impactor, and the divert delta- $V$ to separate the CubeSat impactor is assumed to be an impulsive burn as already discussed. In addition, the mothership's attitude is assumed to immediately reorient to its nominal attitude just after the CubeSat impactor deployment. Actually, reorientation of the mother-ship's attitude may take different durations, dependent on its attitude control strategy, which is certainly another parameter that must be considered at the system design level. For numerical integration, the Runge-Kutta-Fehlberg 7-8th order variable step size integrator is used with truncation error tolerance of $\varepsilon=1 \times 10^{-12}$. The JPL's DE405 is used to derive the accurate planets' ephemeris [41], that is, the Earth and the Sun positions seen from the Moon, with all planetary constants. To convert coordinate systems between the M-MME2000 and M-MMEPM frames, the lunar orientation specified by JPL DE405 is used for high precision work to be performed in the near future.
The current simulation assumes the CubeSat impactor is deployed at the moment when the mother-ship is flying over the north polar region of the Moon with a circular, $90 \mathrm{deg}$ inclined polar orbit of $100 \mathrm{~km}$ altitude. Therefore, initial orbital elements of the mother-ship expressed in the M-MME2000 frame are given as semimajor axis of about $1,838.2 \mathrm{~km}$, zero eccentricity, $90 \mathrm{deg}$ inclination, $0 \mathrm{deg}$ of right ascension of ascending node, and, finally, $90 \mathrm{deg}$ of argument of latitude. As the operational altitude is expected to be $100 \mathrm{~km}$ for Korea's first experimental lunar orbiter mission, the current analysis only considers the $100 \mathrm{~km}$ altitude case. However, additional analysis regarding various mother-ship altitudes could be easily made by simple modifications of the current method. The initial epoch of CubeSat impactor release is assumed to be July 1, 2017, corresponding to Korea's first experimental lunar orbiter mission. Most importantly, the CubeSat impactor release conditions are assumed to be as follows. For release directions, the out-of-plane release direction is increased from $90 \mathrm{deg}$ to $180 \mathrm{deg}$ in steps of $0.5 \mathrm{deg}$, which indicate that the CubeSat impactor will always be deployed in the opposite direction of the mother-ship flight direction, in order to not interfere the mother-ship's original flight path. For in-plane release directions, they are constrained to always have $0 \mathrm{deg}$, to avoid plane changes during the impact phase. Even though the CubeSat is assumed to always be deployed in the opposite direction of the mothership flight direction with 0 deg in-plane release directions, the released CubeSat can be impacted to any location on the lunar surface, as the ground track of the mother-ship will map the entire lunar surface with its assumed 90 deg orbital inclination. For divert delta- $V$ magnitudes, they are increased from $0 \mathrm{~m} / \mathrm{s}$ to $90 \mathrm{~m} / \mathrm{s}$ with $0.5 \mathrm{~m} / \mathrm{s}$ steps. For the closest approach conditions derivation, the convergence criterion is

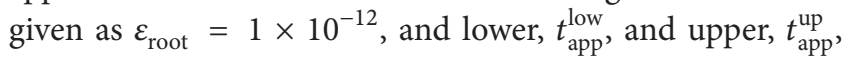
bounds of the time search interval are given to be $0 \mathrm{~min}$ and $118 \mathrm{~min}$, respectively. By applying these constraints, the CubeSat impactor will impact the lunar surface within one orbital period of the mother-ship's orbit (about $118 \mathrm{~min}$ for $100 \mathrm{~km}$ altitude at the Moon), which will ease the communication link problem between the mother-ship and the CubeSat impactor. Also, during the cross range distance computation, the Moon's oblate effect is regarded with flatting coefficient of about $f=0.0012$. During the following discussions, several figures are expressed in normalized units for the ease of interpretation, and all parameters used to normalize units are explained in detail at corresponding subsections. 


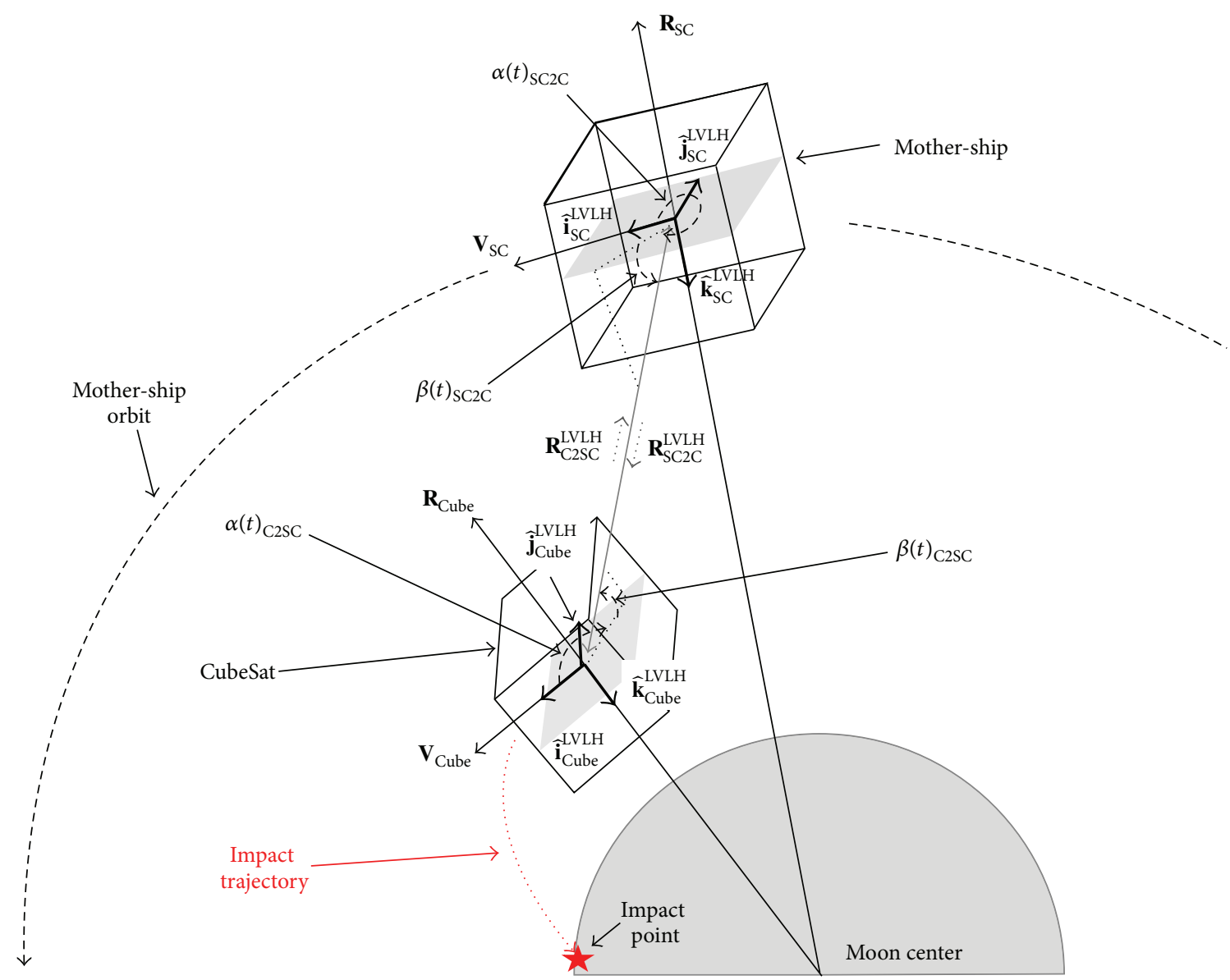

FIGURE 3: Relative geometry between the mother-ship and the CubeSat impactor during the impact phase (not to scale).

\section{Simulation Results}

\subsection{Impact Trajectory Characteristics Analysis}

4.1.1. Impact Opportunities as a Function of Release Conditions. In this subsection, the CubeSat impact opportunities as a function of release conditions are analyzed. The impact opportunities are directly analyzed by using computed closest approach altitudes between the CubeSat impactor and the lunar surface. Corresponding results are depicted in Figure 4. From Figure 4, it can be easily noticed that there are specific of out-of-plane deploy angles and divert delta- $V$ ranges that lead the CubeSat to impact the lunar surface. When the out-ofplane deploy angle is about $180 \mathrm{deg}$, the CubeSat can impact with the minimum amount of divert delta- $V$ magnitude, about $23.5 \mathrm{~m} / \mathrm{s}$. This result indicates that the CubeSat should be released in exactly the opposite direction of the mothership's velocity direction to minimize the required divert delta$V$ magnitude, which is a quite general behavior. However, the minimum magnitude of about $23.5 \mathrm{~m} / \mathrm{s}$ is quite a large amount to be supported only by the P-POD separation mechanism. For example, if performance equivalent to P-POD Mk. III is under consideration, about $21.5 \mathrm{~m} / \mathrm{s}$ of additional divert

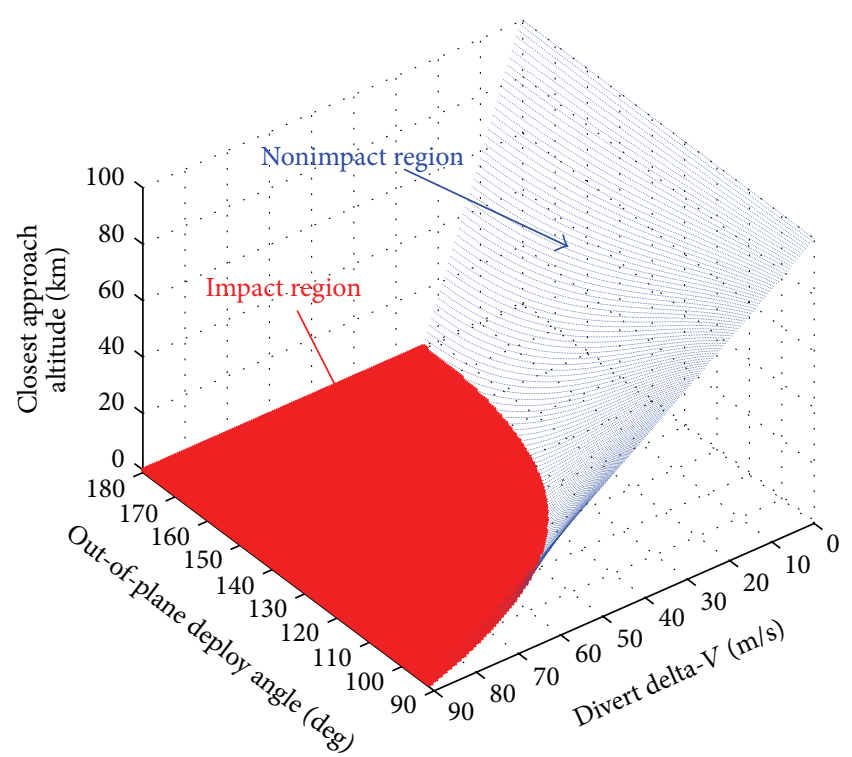

FIGURE 4: CubeSat impact opportunities, when released from a mother-ship having $100 \mathrm{~km}$ altitude and a $90 \mathrm{deg}$ inclined circular orbit around the Moon. 
TABLE 1: List of required divert delta- $V$ s with respect to arbitrary selected out-of-plane deploy angles.

\begin{tabular}{lcc}
\hline $\begin{array}{l}\text { Out-of-plane } \\
\text { deploy angle }(\mathrm{deg})\end{array}$ & $\begin{array}{c}\text { Min. divert } \\
\text { delta- } V(\mathrm{~m} / \mathrm{s})\end{array}$ & $\begin{array}{c}\text { Max. divert } \\
\text { delta- } V(\mathrm{~m} / \mathrm{s})\end{array}$ \\
\hline 90 & More than 90 & N/A \\
100 & 67.50 & 90.00 \\
110 & 51.50 & 90.00 \\
120 & 41.00 & 90.00 \\
130 & 34.00 & 90.00 \\
140 & 29.50 & 90.00 \\
150 & 27.00 & 90.00 \\
160 & 25.00 & 90.00 \\
170 & 24.00 & 90.00 \\
180 & 23.50 & 90.00 \\
\hline
\end{tabular}

delta- $V$ should be supported, since the CubeSat's exit velocity from the P-POD Mk. III is approximately $2.0 \mathrm{~m} / \mathrm{s}$ for a $4 \mathrm{~kg}$ CubeSat [42]. In addition, ejection velocity limitations due to the mother-ship system configuration should additionally be considered.

Another fact discovered is that if the out-of-plane deploy angle is more than $140 \mathrm{deg}$, the rate of degradation in total required divert delta- $V$ magnitude tends to be decreased, that is, only about $6 \mathrm{~m} / \mathrm{s}$ difference on divert delta- $V$ magnitude while about $40 \mathrm{deg}$ of out-of-plane deploy angles are changed (140 deg deploy with about $29.5 \mathrm{~m} / \mathrm{s}$ and 180 deg deploy with about $23.5 \mathrm{~m} / \mathrm{s}$ ). With a maximum magnitude of assumed divert delta- $V, 90 \mathrm{~m} / \mathrm{s}$, the CubeSat can only impact the lunar surface with about $91.5 \mathrm{deg}$ of out-of-plane deploy angle. Thus, if the out-of-plane deploy angle is less than $91.5 \mathrm{deg}$, more than $90 \mathrm{~m} / \mathrm{s}$ of divert delta- $V$ is required. In Table 1 , specific ranges of required divert delta- $V$ magnitude are shown with arbitrary selected out-of-plane deploy angles. Other than these release conditions shown in Table 1 , the CubeSat will not impact the lunar surface and, thus, will require additional analysis with different assumptions on release conditions. For example, if the CubeSat is released with about 180 deg out-of-plane deploy angle with about $2 \mathrm{~m} / \mathrm{s}$ of divert delta- $V$, the CubeSat will not be on course to hit the lunar surface; rather, it will attain an elliptical orbit having about $93.11 \mathrm{~km}$ of perilune altitude (the closest approach distance), which is only about $7 \mathrm{~km}$ of reduced altitude compared to the initial mother-ship's altitude.

4.1.2. Impact Parameter Characteristics. The CubeSat impact parameters during the impact phase are analyzed through this subsection including time left to impact after deploying from the mother-ship, cross range distance, impact angle, and velocity at the time of impact. Among these parameters, the time left to impact after deployment from the mother-ship can be regarded as CFT. In Figure 5, characteristics of derived parameters are shown with release conditions that guaranteed the CubeSat impact. With given assumptions on release conditions, CFTs are found to be within ranges of $15.66 \mathrm{~min}$ (deployed with $130 \mathrm{deg}$ of outof-plane angle with $90 \mathrm{~m} / \mathrm{s}$ divert delta- $V$ ) to $56.00 \mathrm{~min}$ (deployed with $180 \mathrm{deg}$ of out-of-plane angle with $23.5 \mathrm{~m} / \mathrm{s}$ divert delta- $V$ ). For cross range distances, they tend to remain within the range of $1,466.07 \mathrm{~km}$ (deployed with $135.50 \mathrm{deg}$ and $90.00 \mathrm{~m} / \mathrm{s}$ ) to $5,408.80 \mathrm{~km}$ (deployed with $180 \mathrm{deg}$ and $23.50 \mathrm{~m} / \mathrm{s}$ ) and for impact angles they tend to remain within the range of $1.08 \mathrm{deg}$ (deployed with $180 \mathrm{deg}$ and $23.50 \mathrm{~m} / \mathrm{s}$ ) to $3.98 \mathrm{deg}$ (deployed with $135.50 \mathrm{deg}$ and $90.00 \mathrm{~m} / \mathrm{s}$ ). For impact velocities, it is found that they remain within $1.64 \mathrm{~m} / \mathrm{s}$ (deployed with $180 \mathrm{deg}$ and $23.50 \mathrm{~m} / \mathrm{s}$ ) and $1.72 \mathrm{~km} / \mathrm{s}$ (deployed with $91.50 \mathrm{deg}$ and $90.00 \mathrm{~m} / \mathrm{s}$ ), respectively. To compute the impact angle discussed above, cross range distance is computed with the subsatellite point where the CubeSat is released $\left(\lambda\left(t_{r}\right), \phi\left(t_{r}\right)\right)$. Thus, the resultant impact angle could be slightly changed, as it is dependent on the point where the cross ranges are measured, during every moment of the impact phase. The resultant impact angles, 1.08 3.98 deg, sufficiently satisfy the impact angle requirement for the proposed impactor mission, which is given as less than $10 \mathrm{deg}$ [32]. Note that the impact angle condition is constrained for this mission, instead of the duration of time spent over the target area to measure the lunar magnetic field, as the spatial coverage at low altitude is more important than the measurement duration. This is because the onboard magnetometer can measure at a rapid pace to fulfill the science goal. For example, one of the strong candidate impact sites, Reiner Gamma [32, 43, 44], has a spatial extent of about $70 \times 30 \mathrm{~km}$, and when the magnetic field is measured at $200 \mathrm{~Hz}$ with an impact velocity of $\sim 2 \mathrm{~km} / \mathrm{s}$ [32], then the spatial resolution is just $10 \mathrm{~m}$, which is more than sufficient. By analyzing CFTs (shown in Figure 5(a)), the CubeSat's power subsystem requirement, especially battery capacity requirement during the impact phase, could be determined, as the impact is planned to occur on the night side of the Moon to obtain scientifically meaningful data by avoiding solar wind interference [36]. Recall that this simulation is performed under conditions that the CubeSat should impact the lunar surface within one orbital period of the mother-ship's orbit (about $118 \mathrm{~min}$ for $100 \mathrm{~km}$ altitude at the Moon), indicating that the released CubeSat cannot orbit the Moon. This assumption is made to ease the communication architecture design between the mother-ship and the CubeSat impactor. Therefore, it can be easily noticed that the discovered CFTs are all less than $118 \mathrm{~min}$, and also the derived cross range distances are less than about $10,915 \mathrm{~km}$, which is the circumference of the Moon. Although we only considered a mother-ship with an altitude of $100 \mathrm{~km}$, our results revealed several challenging aspects that should be solved in further detailed design studies.

\subsection{Examples of Impact Cases}

4.2.1. Impact Trajectory Analysis. The characteristics of three major representative example impact trajectories, Cases A, $\mathrm{B}$, and $\mathrm{C}$, are analyzed in this subsection. Case A represents the case when the CubeSat is released from a mothership with $130.0 \mathrm{deg}$ of out-of-plane angle with $90.00 \mathrm{~m} / \mathrm{s}$ 


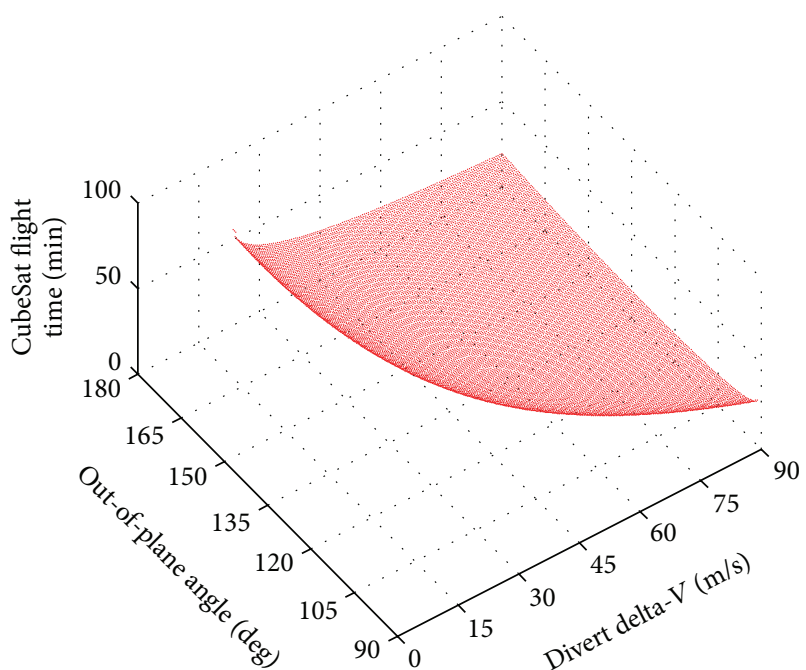

(a)

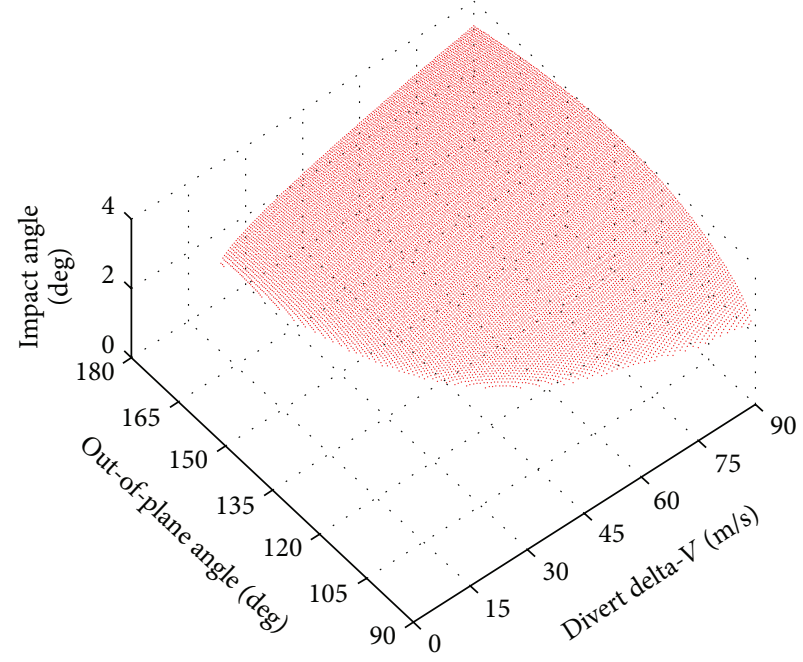

(c)

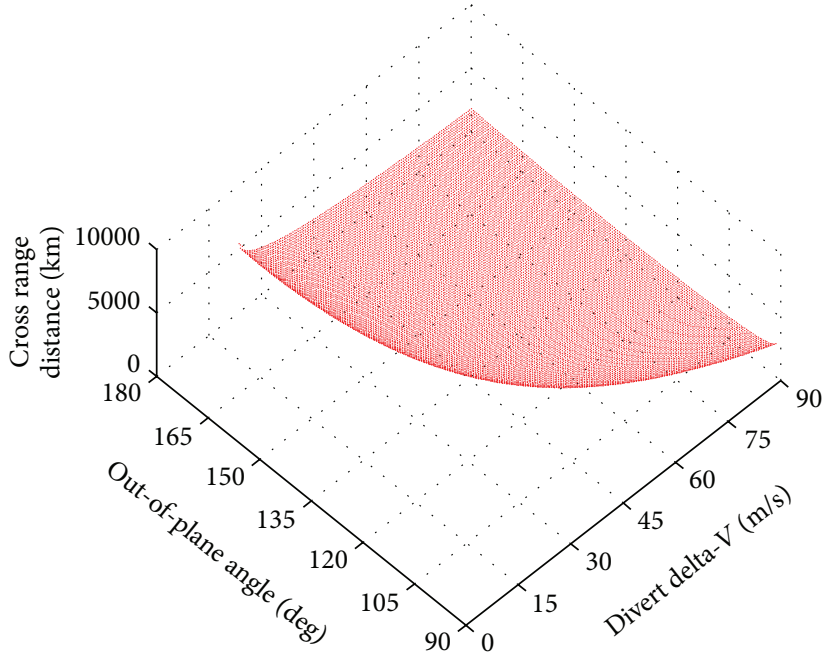

(b)

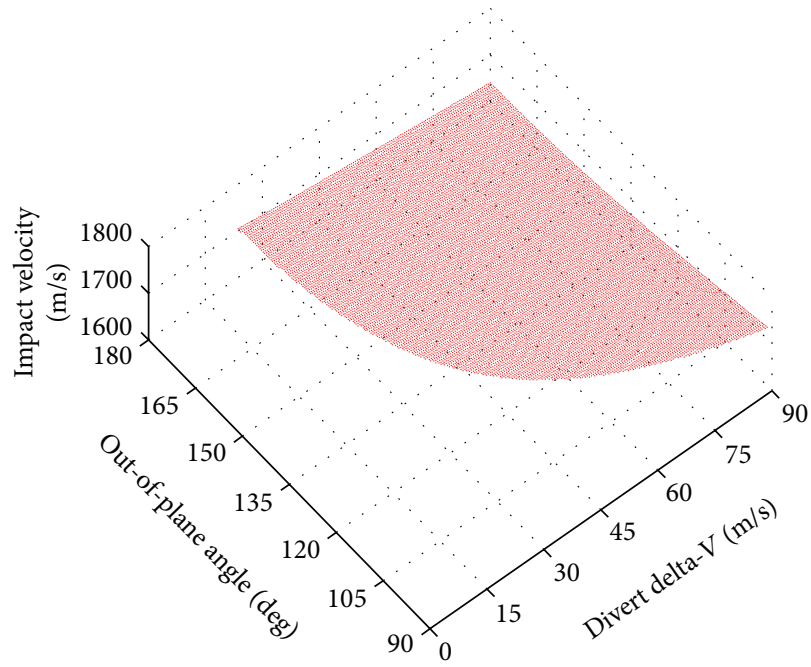

(d)

FIgURE 5: CubeSat impact parameter characteristics during the impact phase. (a) CubeSat flight time, (b) cross range distance, (c) impact angle, and (d) impact velocity.

divert delta- $V$, Case $\mathrm{B}$ is the case released with $164.50 \mathrm{deg}$ with $31.50 \mathrm{~m} / \mathrm{s}$ of divert delta- $V$, and, finally, Case $C$ is the case released with 180.00 deg of out-of-plane angle with $23.50 \mathrm{~m} / \mathrm{s}$ divert delta- $V$, respectively. Note that Cases A and $\mathrm{C}$ are the minimum (about $15.66 \mathrm{~min}$ ) and maximum (about $55.92 \mathrm{~min}$ ) CFT cases out of the entire simulation cases, and Case B is selected as an example which has $35.83 \mathrm{~min}$ of CFT, the average CFT between Cases A and C. Actually, among all solutions, there were three different cases having $35.83 \mathrm{~min}$ of CFT with different release conditions, $164.50 \mathrm{deg}$ with $31.50 \mathrm{~m} / \mathrm{s}, 150.50 \mathrm{deg}$ with $32.00 \mathrm{~m} / \mathrm{s}$, and $139.00 \mathrm{deg}$ with $34.00 \mathrm{~m} / \mathrm{s}$. Among these three cases, the case with minimum divert delta- $V$ with $31.50 \mathrm{~m} / \mathrm{s}$ is selected for Case B. In Figure 6, associated impact trajectories are shown with normalized distance units, Lunar Unit (LU), where $1 \mathrm{LU}$ is about $1,738.2 \mathrm{~km}$. In addition, Cases $\mathrm{A}, \mathrm{B}$, and $\mathrm{C}$ shown in
Figure 6 can be understood as short-, medium-, and long-arc cases, respectively.

For Case A, the CubeSat is released at June 1, 2017, 00:00:00 (UTC) and impact occurred at June 1, 2017, 00:15:40 (UTC). At the time of the CubeSat release, the mother-ship's velocity is found to be about $1.63 \mathrm{~km} / \mathrm{s}$ and the CubeSat impacted with about $1.67 \mathrm{~km} / \mathrm{s}$. For Cases B and C, both the CubeSat release time and mother-ship's velocity at the time of release are the same as with Case A, but the impact time is found to be June 1, 2017, 00:35:50 (UTC) with an impact velocity of about $1.69 \mathrm{~km} / \mathrm{s}$ for Case B. For Case C, the impact time is found to be about June 1, 2017, 00:55:58 (UTC) with an impact velocity of about $1.70 \mathrm{~km} / \mathrm{s}$. As expected, although less divert delta- $V$ was applied $(90.00 \mathrm{~m} / \mathrm{s}$ for Case A, $31.50 \mathrm{~m} / \mathrm{s}$ for Case B, and $23.50 \mathrm{~m} / \mathrm{s}$ for Case C), a faster impact velocity is achieved as CFT becomes longer; that is, 


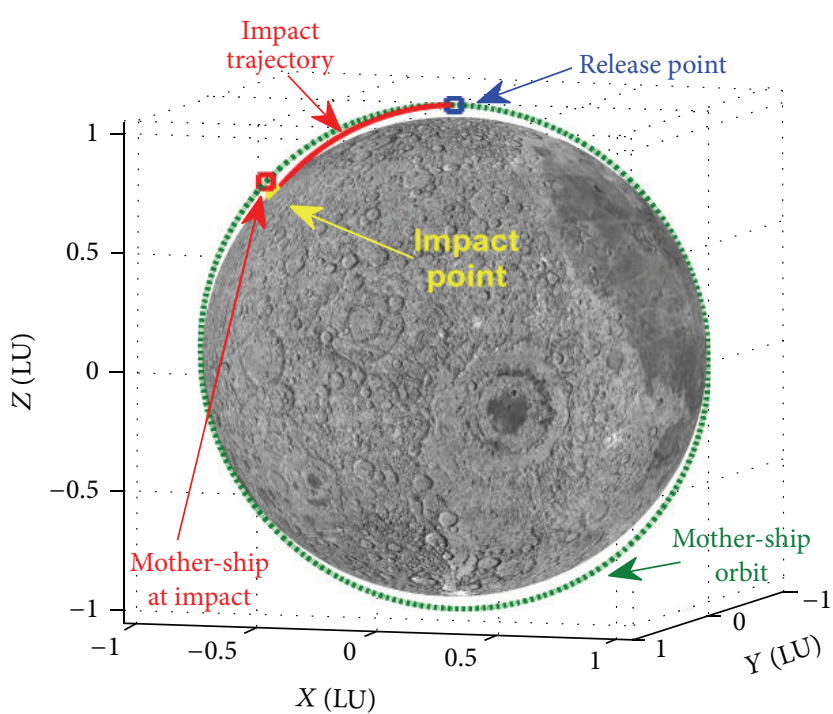

(a)

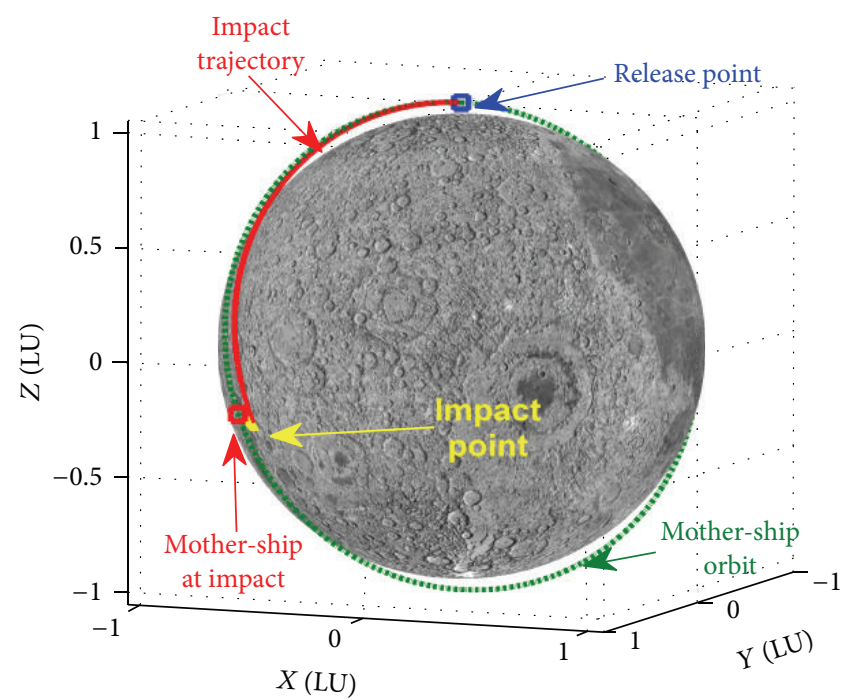

(b)

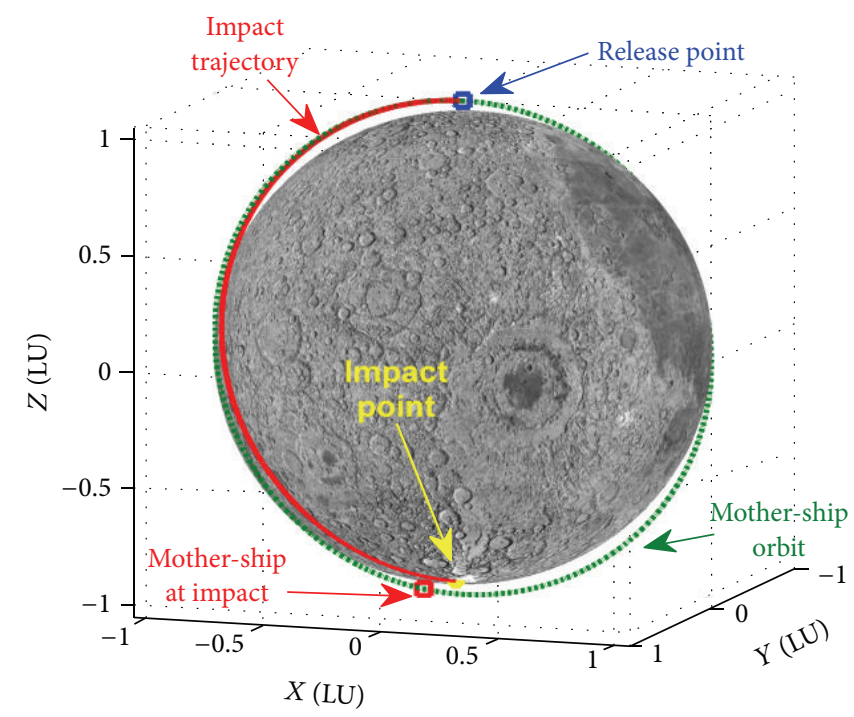

(c)

Figure 6: Selected example of CubeSat impact trajectories; (a) is for Case A, (b) is for Case B, and (c) is for Case C.

Case $C$ is about $30 \mathrm{~m} / \mathrm{s}$ faster than Case A as the CubeSat is more accelerated. In addition, for the same reasons, we discovered greater separations on relative ground track positions between the mother-ship and the CubeSat impactor near the time of impact. By analyzing the CFT, the impact location can roughly be estimated. For example, for Case C, the impact location will be near the south pole of the Moon, as the derived CFT is almost half of the mother-ship's orbital period (about $118 \mathrm{~min}$ ) as shown in Figure 6(c). When only regarding the divert delta- $V$ 's magnitude, which still requires additional support from an onboard thruster to contribute the remainder of the delta- $V$, it seems that Case $\mathrm{C}$ would be the appropriate choice for the proposed impact mission. However, if the CubeSat power availability is considered as the major design driver, Case B would be the proper choice, which has a CFT of about $35.83 \mathrm{~min}$. This is due to the fact that the current design for the CubeSat impactor mission considers operation only with a charged battery (current power subsystem is expected to support maximum of about $30 \mathrm{~min}$ ) during the impact phase. However, Case B still requires more divert delta- $V$, which is another critical aspect that has to be satisfied by means of sustained system design studies.

In Figure 7, ground tracks for the selected three CubeSat impact trajectories are shown. In Figure 7, as already discussed, it can be clearly seen that the relative ground locations between the mother-ship and the CubeSat impactor broaden at the time of impact with longer CFTs. The positions of the Earth and Sun at the time of impact are also depicted in Figure 7, which could aid in further detailed mission studies, 


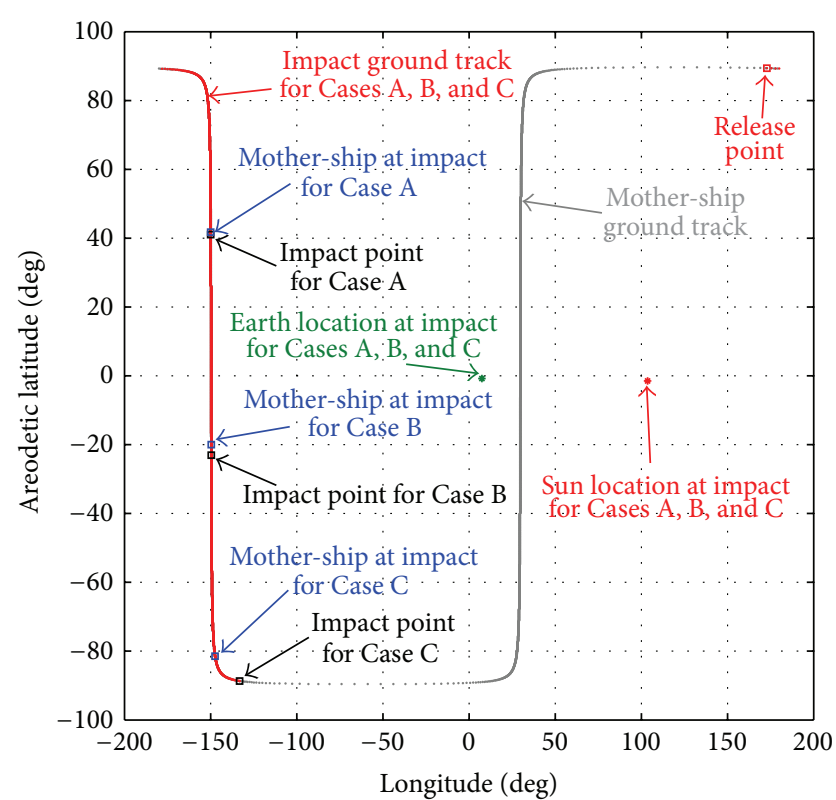

Figure 7: Ground tracks for the selected CubeSat impact trajectories, Cases A, B, and C with positions of the Sun and Earth at the impact time.

that is, the Earth communication opportunities for the mother-ship during the impact phase as well as determining whether the impact would occur on the day or night side. It is expected that further detailed analysis could be easily made based on the current analysis.

4.2.2. Impact Angle Analysis. In this subsection, impact angle variations during the impact phase for three different example cases (Cases A, B, and C) are analyzed. Before discussing impact angle variations, the CubeSat altitude variations during the impact phase are firstly analyzed as shown in Figure $8(\mathrm{a})$. In Figure $8(\mathrm{a})$, the $x$-axis denotes normalized remaining cross range distance before the impact, and the $y$ axis represents areodetic altitude for each case. In Figure 8(a), cross range distance is normalized with $1,471.35 \mathrm{~km}$ for Case A, $3,411.77 \mathrm{~km}$ for Case B, and $5,404.29 \mathrm{~km}$ for Case C, respectively. To normalize areodetic altitudes for every case, $102.09 \mathrm{~km}$ is used. As expected, areodetic altitude almost linearly decreases as cross range approaches zero for Case A. However, for Cases B and C, areodetic altitudes do not linearly decrease but decrease with small fluctuations when compared to Case A. The main cause of this phenomenon is due to the shape of the resultant CubeSat impact trajectory. For example, if impact had not occurred near perilune for Case $\mathrm{C}$, then the resultant impact trajectory would be considered an elliptical orbit around the Moon, and, thus, the depicted behaviors of altitude variations for given impact trajectories (Cases B and C) are rather general results.

Based on altitude variations (shown in Figure 8(a)), the impact angle variations are analyzed as shown in Figure 8(b). In Figure $8(\mathrm{~b})$, the $x$-axis indicates the remaining time to impact the lunar surface expressed in normalized time units, and the $y$-axis indicates the CubeSat impact angle expressed in deg. To normalize time units, $15.66 \mathrm{~min}$ is used for Case A, $35.83 \mathrm{~min}$ for Case B, and $55.97 \mathrm{~min}$ for Case C, respectively. At the beginning of the impact phase, at the CubeSat release time, the impact angle for Case $\mathrm{A}$ is found to be about $3.97 \mathrm{deg}$, for Case B about $1.72 \mathrm{deg}$, and for Case C about $1.08 \mathrm{deg}$, respectively. The impact angle increases linearly to about $4.66 \mathrm{deg}$ for Case A; however, not surprisingly, different trends are observed for Cases B and C. For Case $\mathrm{B}$, the impact angle increases to about $2.09 \mathrm{deg}, 19.47 \mathrm{~min}$ after release, and decreases linearly for the remainder of the impact phase (ending at about $1.75 \mathrm{deg}$ at impact). For Case $\mathrm{C}$, the impact angle increases to about $1.21 \mathrm{deg}, 13.78 \mathrm{~min}$ after the CubeSat release, and decreases to about $0.05 \mathrm{deg}$ at the final impact time. Areodetic altitudes achieved by the CubeSat before about $10 \mathrm{~km}$ cross range distance apart from the impact point are as follows: about $819.91 \mathrm{~m}$ for Case A, about $308.33 \mathrm{~m}$ for Case $\mathrm{B}$, and only about $8 \mathrm{~m}$ for Case $\mathrm{C}$ are derived, respectively. If the achieved impact angle is considered as the major design driver, Case A would be a better option than Case B or C, as it achieves higher impact angle during the impact phase since there is uncertainty in lunar surface heights. Indeed, the resultant relations between divert delta- $V$ magnitude and impact angle shown through this subsection would be another major issue to be dealt with in further detailed trade-off design studies. In Table 2, detailed mission parameters obtained for three different cases are summarized. Note that every eastern longitude and every northern latitude shown in Table 2 is based on M-MMEPM frame, and associated altitude and cross range distances are all in an areodetic reference frame.

4.2.3. Relative Motion Analysis. During the impact phase, relative motion between the mother-ship and the CubeSat impactor is one of the major factors that has to be analyzed for the communication architecture design. In Figure 9, relative motion characteristics are shown for three different impact cases. $x$-axes in subfigures of Figure 9 are expressed in normalized time units, and $y$-axes indicate in-plane (left side of Figure 9) and out-of-plane (right side of Figure 9) direction angles in deg. To normalize time units, $15.66 \mathrm{~min}$ is used for Case A (shown at Figures 9(a) and 9(b)), 35.83 min for Case B (shown at Figures 9(c) and 9(d)), and $55.92 \mathrm{~min}$ for Case C (shown at Figures 9(e) and 9(f)), respectively. In addition, solid lines represent relative locations of the mother-ship seen from the CubeSat ("C to $\mathrm{M}$ " in the following discussions) and dotted lines represent the location of the CubeSat seen from the mother-ship (" $\mathrm{M}$ to $\mathrm{C}$ " in the following discussions). By investigating in-plane direction motions (left side of the Figure 9) variations, it is observed that a "phase shift" between the mother-ship and the CubeSat occurred during the impact phase in every simulated case. As an example of Case A, inplane angle remained to be about $180 \mathrm{deg}$ (for $M$ to $C$ ) or $0 \mathrm{deg}$ (for $\mathrm{C}$ to $\mathrm{M}$ ) at the early phase of the CubeSat release and then switched to be $0 \mathrm{deg}$ (for $M$ to $C$ ) and $180 \mathrm{deg}$ (for $\mathrm{C}$ to $\mathrm{M}$ ) for the remaining time of impact phase. This result indicates that the mother-ship will fly ahead of the CubeSat if seen from the CubeSat itself or will fly behind the mother-ship if seen from the mother-ship during about 11.93 min (herein after the phase I). Then, the "phase shift" 
TABLE 2: Detailed mission parameters derived for Cases A, B, and C.

\begin{tabular}{|c|c|c|c|}
\hline Parameters & Short-arc case (Case A) & Medium-arc case (Case B) & Long-arc case (Case C) \\
\hline Release time & 2017-06-01 00:00:00 (UTC) & $\leftarrow$ & $\leftarrow$ \\
\hline Impact time & 2017-06-01 00:15:40 (UTC) & 2017-06-01 00:35:50 (UTC) & 2017-06-01 00:55:58 (UTC) \\
\hline Mother-ship velocity at release & $1.63(\mathrm{~km} / \mathrm{s})$ & $\leftarrow$ & $\leftarrow$ \\
\hline CubeSat velocity at impact & $1.67(\mathrm{~km} / \mathrm{s})$ & $1.69(\mathrm{~km} / \mathrm{s})$ & $1.70(\mathrm{~km} / \mathrm{s})$ \\
\hline CubeSat time of flight & $15.66(\min )$ & $35.83(\min )$ & $55.92(\min )$ \\
\hline \multicolumn{4}{|l|}{ Release location } \\
\hline Longitude & $172.85(\mathrm{deg})$ & $\leftarrow$ & $\leftarrow$ \\
\hline Latitude & 89.39 (deg) & $\leftarrow$ & $\leftarrow$ \\
\hline Altitude & $102.09(\mathrm{~km})$ & $\leftarrow$ & $\leftarrow$ \\
\hline \multicolumn{4}{|l|}{ Impact location } \\
\hline Longitude & $-149.92(\mathrm{deg})$ & $-149.62(\mathrm{deg})$ & $-133.14(\mathrm{deg})$ \\
\hline Latitude & $41.03(\mathrm{deg})$ & $-23.06(\mathrm{deg})$ & $-88.70(\mathrm{deg})$ \\
\hline Altitude & $0.00(\mathrm{~km})$ & $\leftarrow$ & $\leftarrow$ \\
\hline \multicolumn{4}{|l|}{ Mother-ship at impact } \\
\hline Longitude & $-149.93(\mathrm{deg})$ & $-149.64(\mathrm{deg})$ & $-147.44(\mathrm{deg})$ \\
\hline Latitude & 41.73 (deg) & $-19.99(\mathrm{deg})$ & $-81.48(\mathrm{deg})$ \\
\hline Altitude & $100.92(\mathrm{~km})$ & $100.24(\mathrm{~km})$ & $102.04(\mathrm{~km})$ \\
\hline \multicolumn{4}{|l|}{ Earth at impact } \\
\hline Longitude & $7.44(\mathrm{deg})$ & $7.44(\mathrm{deg})$ & $7.44(\mathrm{deg})$ \\
\hline Latitude & $-0.76(\mathrm{deg})$ & $-0.80(\mathrm{deg})$ & $-0.83(\mathrm{deg})$ \\
\hline \multicolumn{4}{|l|}{ Sun at impact } \\
\hline Longitude & $103.62(\mathrm{deg})$ & $103.44(\mathrm{deg})$ & $103.27(\mathrm{deg})$ \\
\hline Latitude & $-1.50(\mathrm{deg})$ & $-1.50(\mathrm{deg})$ & $-1.50(\mathrm{deg})$ \\
\hline Cross distance range (release to impact) & $1,471.35(\mathrm{~km})$ & $3,411.77(\mathrm{~km})$ & $5,404.29(\mathrm{~km})$ \\
\hline \multicolumn{4}{|l|}{ Impact angle } \\
\hline At release & $3.97(\mathrm{deg})$ & $1.72(\mathrm{deg})$ & $1.08(\mathrm{deg})$ \\
\hline At about $10 \mathrm{~km}$ cross range distance before impact & $4.66(\mathrm{deg})$ & $1.86(\mathrm{deg})$ & $0.04(\mathrm{deg})$ \\
\hline
\end{tabular}

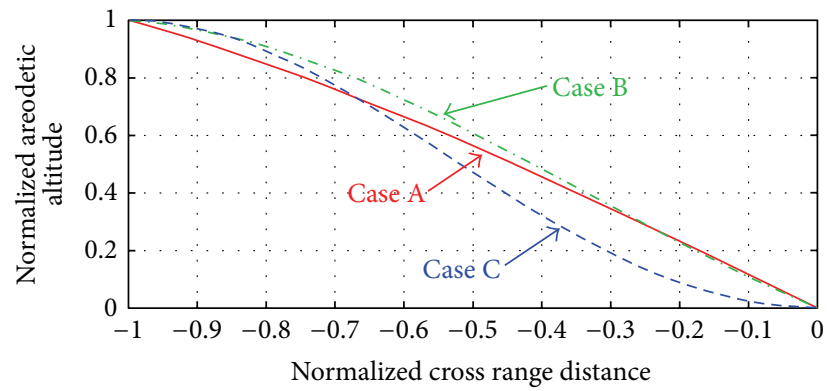

(a)

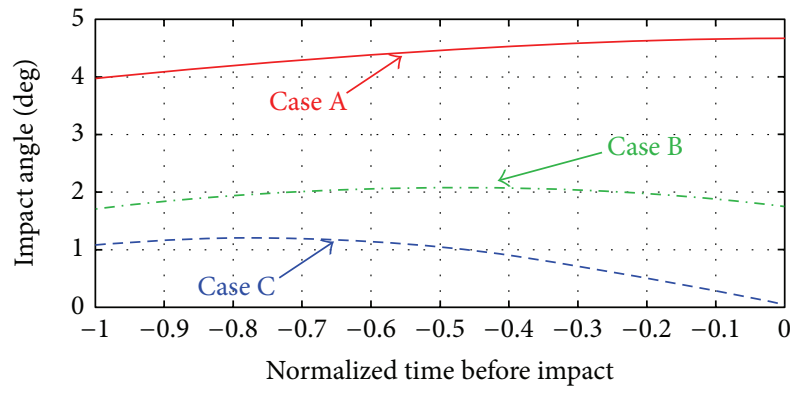

(b)

Figure 8: Altitude (a) and impact angle (b) variations and during the impact phase for Cases A, B, and C.

occurs, which indicates the relative flight position is changed during the remainder of time (herein after the phase II), about $3.73 \mathrm{~min}$. Thus, about $76.18 \%$ of the entire impact phase will be phase I for Case A. For Case B, phase I is found to be about $20.16 \mathrm{~min}$ and $10.67 \mathrm{~min}$ for phase II, such that the phase I portion is about $65.39 \%$. Finally, for Case C, phase $\mathrm{I}$ is found to be about $23.89 \mathrm{~min}$ and $32.08 \mathrm{~min}$ for phase II, with the phase I portion equal to $42.68 \%$. These results indicate that as the CFT gets longer, the portion of phase I will be less (or phase II will be more) due to the dynamic behavior of the CubeSat in the impact phase as previously discussed in Section 4.2.1. For out-of-plane direction relative motions, similar behaviors were obtained. The existence of the phase shift could also be confirmed through the sign of rate changes at the peaks of out-of-plane direction variations, about $-89.99 \mathrm{deg}$ for $\mathrm{C}$ to $\mathrm{M}$ and about $89.99 \mathrm{deg}$ for $M$ to $C$ as shown in Figures $9(\mathrm{~b}), 9(\mathrm{~d})$, and 9(f). Note that the out-of-plane direction angle for $\mathrm{C}$ to $\mathrm{M}$ 


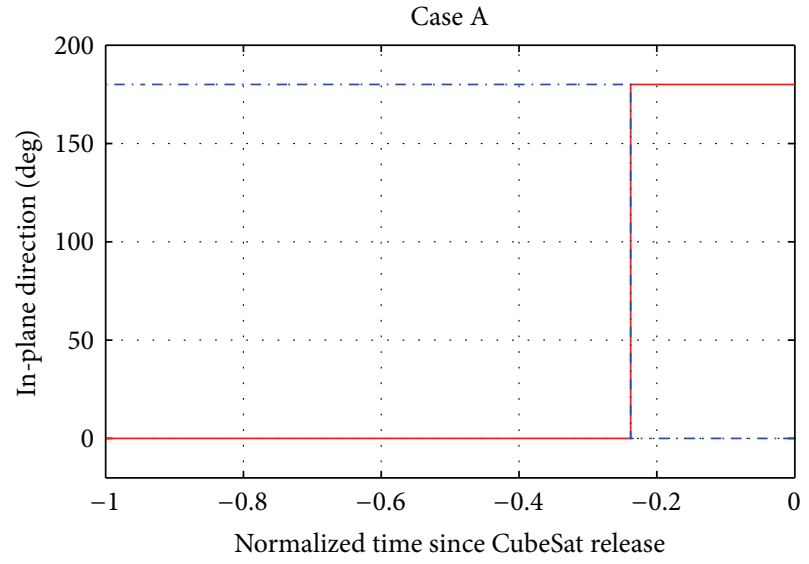

(a)

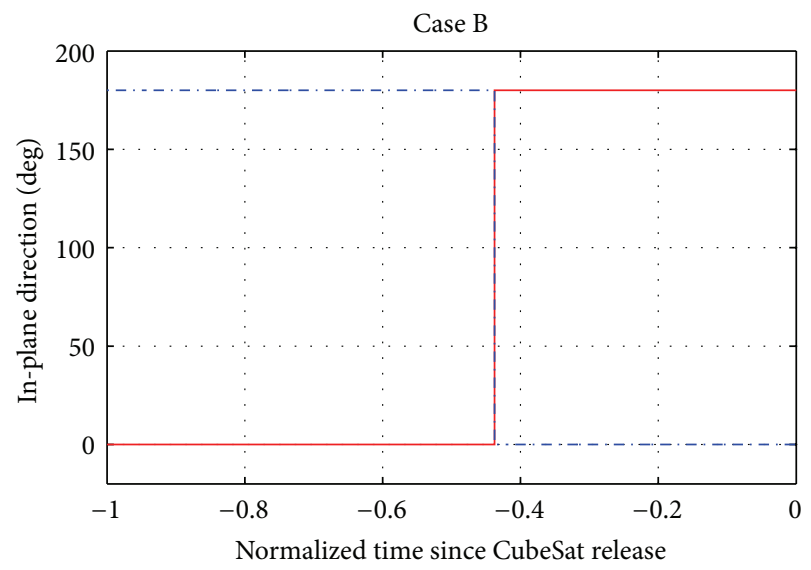

(c)

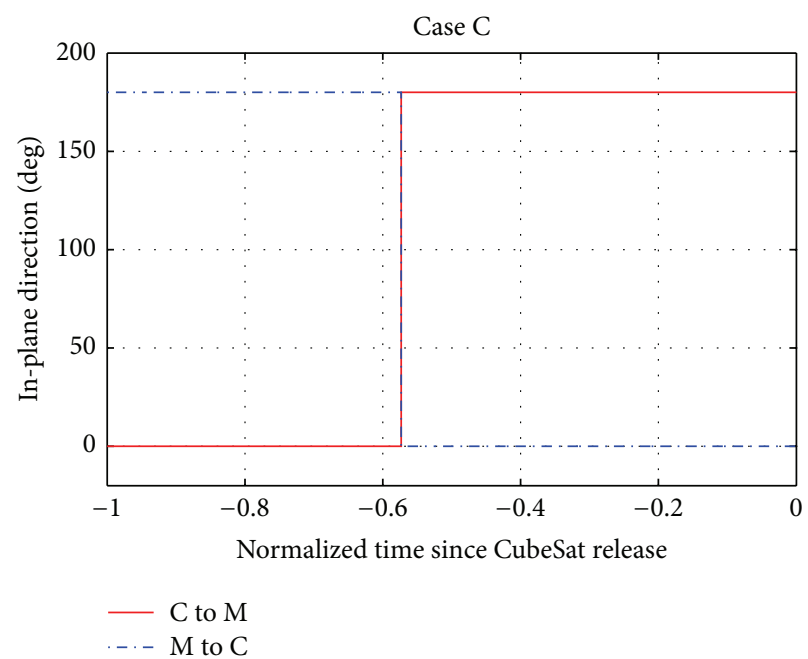

(e)

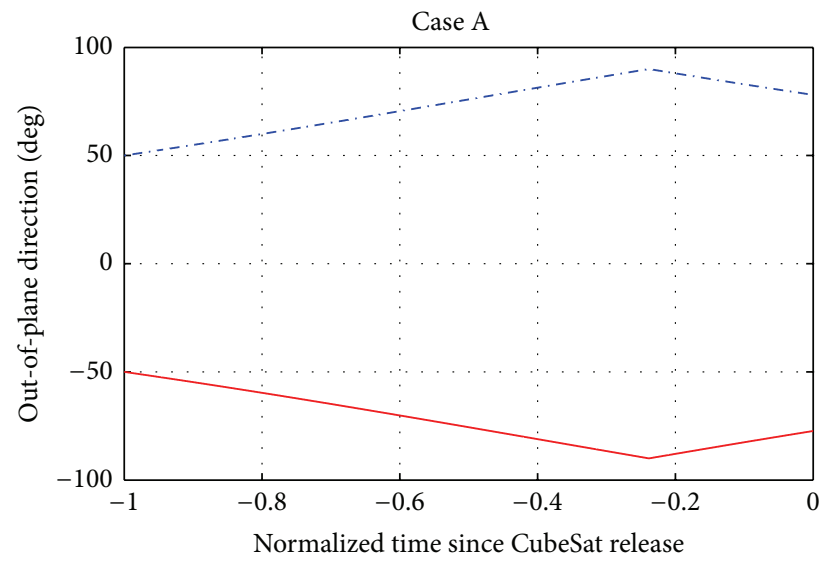

(b)

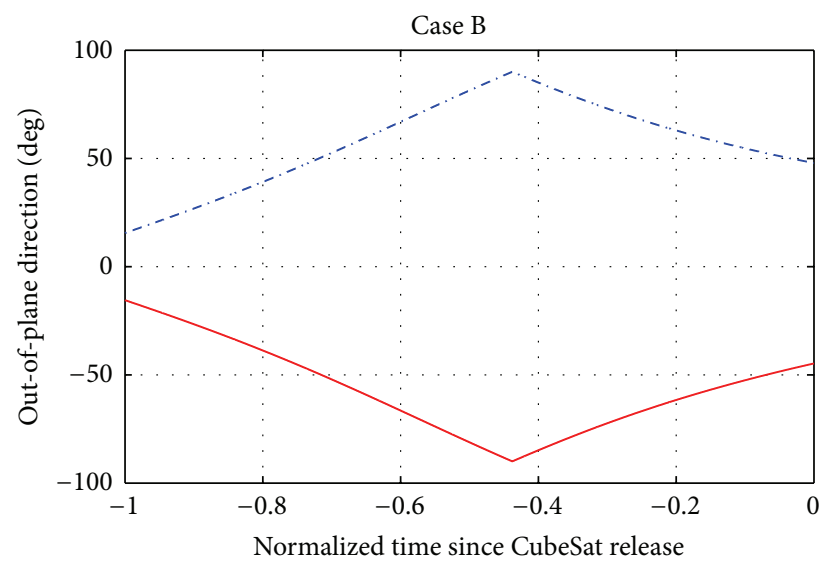

(d)

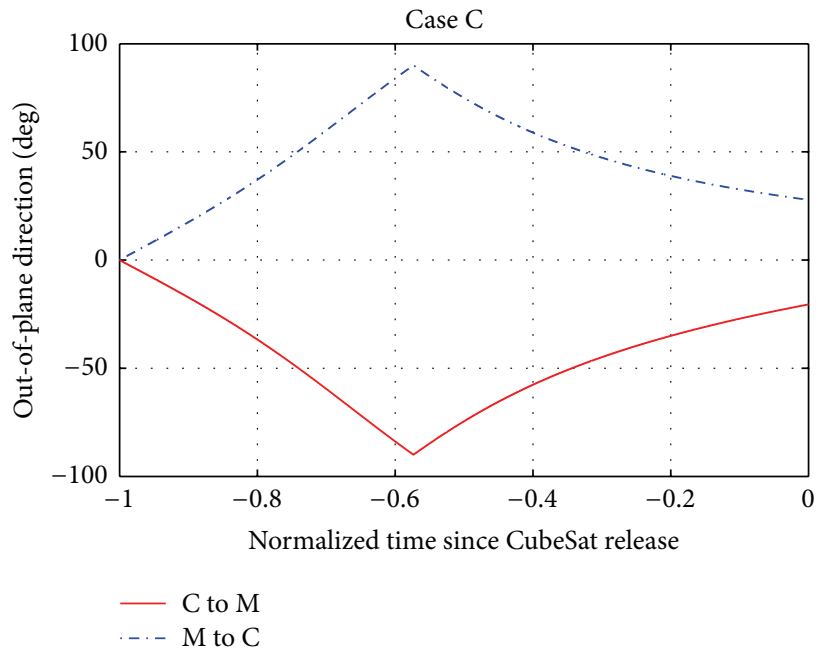

(f)

FIGURE 9: Relative motion between the mother-ship and CubeSat during impact phase. In-plane (left) and out-of-plane (right) direction angle variations for Case A ((a) and (b)), Case B ((c) and (d)), and Case C ((e) and (f)). 


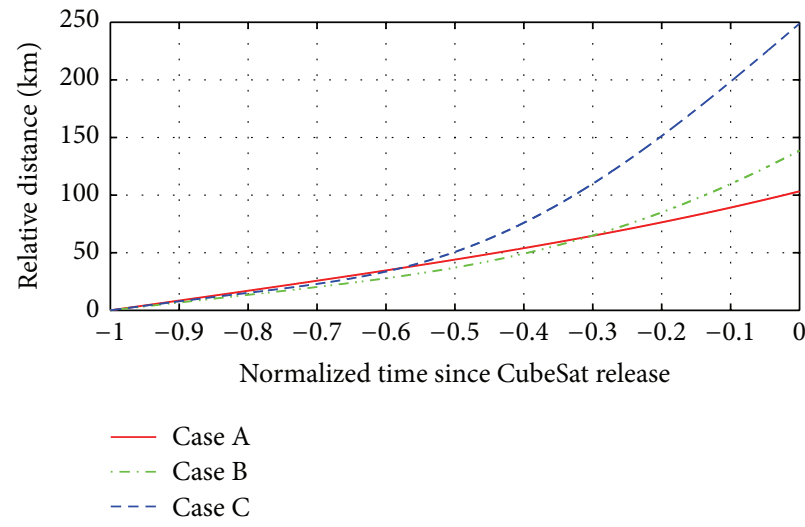

(a)

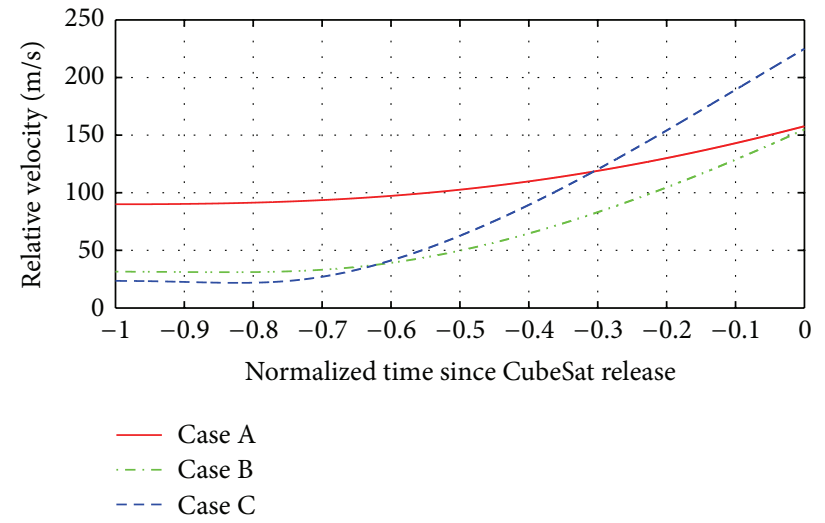

(b)

FIGURE 10: Relative range (a) and velocity (b) variation histories between the mother-ship and the CubeSat during the three different (Cases $\mathrm{A}, \mathrm{B}$, and C) impact cases.

is always negative, as the mother-ship will always be located above the defined local horizontal plane of the CubeSat frame. As expected, the initial value of the out-of-plane direction angle variations is directly related to the out-of-plane CubeSat release direction. However, as analyses in this study are made under the assumption of instantaneous attitude reorientation of the mother-ship after CubeSat separation, the results shown in this subsection may vary based on the mother-ship's attitude control strategy, especially for out-of-plane relative directions. For additional analysis, relative range and velocity variations between the mother-ship and CubeSat during the impact phase are investigated for three different example cases as shown in Figure 10. Note that variation histories shown in Figure 10 are all $\mathrm{C}$ to $\mathrm{M}$ cases. Without a doubt, relative distances between the mother-ship and CubeSat increase as time reaches the impact time, and Case $\mathrm{C}$ showed the largest separation distance at the time of impact, about $248.82 \mathrm{~km}$, than the other two cases, about $103.29 \mathrm{~km}$ for Case $\mathrm{A}$ and $138.66 \mathrm{~km}$ for Case B. For relative velocity variations, as expected, the final relative velocity of Case $\mathrm{C}$ showed the maximum, about $224.91 \mathrm{~m} / \mathrm{s}$, with the largest difference between the initial release and final relative velocity at impact. For Case A, the final relative velocity between the mothership and the CubeSat is found to be about $154.91 \mathrm{~m} / \mathrm{s}$ and $157.81 \mathrm{~m} / \mathrm{s}$ for Case B. Results provided in this subsection are expected to be used as a basis for the detailed communication system design, that is, optimum onboard antenna location to maximize the communication performance between the two satellites during the impact phase.

4.3. Further Analysis Planned. As the current study is performed as a part of early system design activities, a future study will be carried out for more detailed mission analysis. To provide enough divert delta- $V$ magnitude, alternative approaches will be taken into account, such as use of a miniaturized CubeSat thruster during the impact phase to compensate the insufficient delta- $V$ from P-POD, separation at a lower altitude, as well as with enhanced P-POD delta- $V$ performance that we can possibly achieve while satisfying the requirement on shallow impact angle. Therefore, numerous trade-off studies will be made in further analyses. During the numerous trade-off studies, the effect of the perturbing forces due to the nonsphericity of the Moon and 3rd bodies (e.g., the Earth) to the CubeSat impact trajectory will also be analyzed in detail. The optimization of impact trajectory and attitude control strategy of the CubeSat impactor will also be considered. Additionally, analysis with a specified target (i.e., Reiner Gamma) impact area and additional diagnostics of the mother-ship's orbital elements at the time of CubeSat release will be performed. Most importantly, tolerable deploy delta- $V$ errors (not only the magnitude but also the directions) to land in an ellipse of a given target area will be analyzed. Through this analysis, the minimum requirements on the CubeSat onboard propulsion system and numerous insights into the mother-ship's orbit and attitude determination accuracy can be obtained. By regarding allowable delta- $V$ errors of the CubeSat onboard propulsion system and P-POD mechanism, we could also place some constraints on the CubeSat release time. For example, only small delta- $V$ errors will be accepted if the CubeSat is released very early before impact; in contrast, relatively large delta- $V$ errors are permitted if the CubeSat is released very close to the impact time to achieve the required impact accuracy. In addition, to correct delta- $V$ errors, separation of the deorbit burn strategy into 2 3 stages or more could be regarded during the detailed mission design phase. Although several challenging aspects remain and still require sustained research, preliminary analysis results obtained from this study will give numerous insights into the design field of planetary impactor missions with CubeSatbased payloads.

\section{Conclusions}

As a part of preliminary mission design and analysis activities, the trajectory characteristics of a lunar CubeSat impactor released from a lunar orbiter, a mother-ship, are analyzed in this study. The mother-ship is assumed to have a circular polar orbit with an inclination of 90 degrees at a $100 \mathrm{~km}$ 
altitude at the Moon. Two release conditions are applied to separate the CubeSat, the eject direction (in-plane and out-of-plane with respect to mother-ship's LVLH frame) and the divert delta- $V$ 's magnitude, as these are major factors that determine the flight path of the CubeSat impactor. As a result, the CubeSat impact opportunities are analyzed with related mission parameters: appropriate release directions, divert delta- $V$ s magnitude, CubeSat flight times, impact velocities, cross ranges, and impact angles. In addition, the relative flight motion between the mother-ship and the CubeSat during the impact phase is analyzed to support detailed communication system design activities. It is found that the lunar impactor and its trajectory characteristics strongly depend on the divert delta- $V$ magnitude rather than the applied release directions. Within the release conditions that we have assumed, the CubeSat flight time after separation takes about $15.66 \sim 56.00 \mathrm{~min}$, with about $1.64 \sim 1.72 \mathrm{~km} / \mathrm{s}$ impact velocity. Also, the cross range (travel distance) on a lunar ground track was found to be about 1,466.07 5,408.80 km, with an impact angle of about 1.08 3.98 deg. It is confirmed that the very shallow impact angle (less than $10 \mathrm{deg}$ ) can be achieved with the proposed CubeSat impactor release scenarios, which is a critical requirement to meet the science objectives. However, the required minimum divert delta- $V$ magnitude to impact the CubeSat is found to be $23.5 \mathrm{~m} / \mathrm{s}$, and this is quite large compared to the capabilities of the current available P-POD system. From relative motion analysis, it is found that there is a phase-shift stage between the mother-ship and the CubeSat during the impact phase, and the moment of this phase-shift is strongly dependent on the CubeSat flight time. This indicates that the onboard antenna locations should be optimized to maximize the communication performance within limited power sources during the impact phase. Although this analysis is made using basic dynamics and several assumptions, additional guidelines for further mission design and analysis are well defined from the current results and a future study will be carried out for more detailed mission analysis. Also, it is expected that the analysis methods described in this work can easily be modified and applied to any other similar future planetary impactor missions with CubeSat-based payloads.

\section{Conflict of Interests}

The authors declare that there is no conflict of interests regarding the publication of this paper.

\section{Acknowledgments}

This work was supported by the BK21 Plus and NRF2014M1A3A3A02034761 Program through the National Research Foundation (NRF) funded by the Ministry of Education and the Ministry of Science, ICT and Future Planning of South Korea.

\section{References}

[1] M. Swartwout, "The first one hundred CubeSats: a statistical look," Journal of Small Satellites, vol. 2, no. 2, pp. 213-233, 2013.
[2] A. T. Klesh and J. C. Castillo-Rogez, "Applications of NanoSats to planetary exploration," in Proceedings of the AIAA SPACE Conference \& Exposition, Pasadena, Calif, USA, September 2012.

[3] R. L. Staehle, D. Blaney, H. Hemmati et al., "Interplanetary CubeSats: opening the solar system to a broad community at lower cost," Journal of Small Satellites, vol. 2, no. 1, pp. 161-186, 2013.

[4] D. L. Blaney, R. L. Staehle, B. Betts et al., "Interplanetary CubeSats: small, low cost missions beyond low Earth Orbit," in Proceedings of the 43rd Lunar and Planetary Science Conference, p. 1868, Woodlands, Tex, USA, March 2012.

[5] J. Vannitsen, B. Segret, J. J. Maiu, and J.-C. Juang, "CubeSat on Earth-Mars Free Return Trajectory to study radiation hazards in the future manned mission," in Proceedings of the European Planetary Science Congress (EPSC '13), EPSC2013-1088, London, UK, September 2013.

[6] E. Asphaug and J. Thangavelautham, "Asteroid regolith mechanics and primary accretion experiments in a CubeSat," in Proceedings of the 45th Lunar and Planetary Science Conference, p. 2306, Woodlands, Tex, USA, March 2014.

[7] Y. J. Song, S. Y. Park, K. H. Choi, and E. S. Sim, “Development of Korean preliminary lunar mission design software," Journal of The Korean Society for Aeronautical \& Space Sciences, vol. 36, no. 4, pp. 357-367, 2008.

[8] Y. J. Song, S. Y. Park, K. H. Choi, and E. S. Sim, "Optimal earthmoon trajectory design using constant and variable low thrust," Journal of the Korean Society for Aeronautical Space Sciences, vol. 37, no. 9, pp. 843-854, 2009.

[9] Y.-J. Song, S.-Y. Park, K.-H. Choi, and E.-S. Sim, "A lunar cargo mission design strategy using variable low thrust," Advances in Space Research, vol. 43, no. 9, pp. 1391-1406, 2009.

[10] Y. J. Song, J. Woo, S. Y. Park, K. H. Choi, and E. S. Sim, “The earth moon transfer trajectory design and analysis using intermediate loop orbits," Journal of Astronomy and Space Sciences, vol. 26, no. 2, pp. 171-186, 2009.

[11] J. Woo, Y.-J. Song, S.-Y. Park, H.-D. Kim, and E.-S. Sim, “An earth-moon transfer trajectory design and analysis considering spacecraft's visibility from daejeon ground station at TLI and LOI maneuvers," Journal of Astronomy and Space Science, vol. 27, no. 3, pp. 195-204, 2010.

[12] Y. J. Song, S. Y. Park, H. D. Kim, J. H. Lee, and E. S. Sim, “Trans Lunar Injection (TLI) maneuver design and analysis using finite thrust," Journal of The Korean Society for Aeronautical \& Space Sciences, vol. 38, no. 10, pp. 998-1011, 2010.

[13] T. S. No and G. E. Jeon, "A study on optimal earth-moon transfer orbit design using mixed impulsive and continuous thrust," Journal of the Korean Society for Aeronautical \& Space Sciences, vol. 38, no. 7, pp. 684-692, 2010.

[14] D. H. Lee and H. C. Bang, "Low thrust, fuel optimal earth escape trajectories design," Journal of the Korean Society for Aeronautical \& Space Sciences, vol. 35, no. 7, pp. 647-654, 2007.

[15] Y.-J. Song, S.-Y. Park, H.-D. Kim, J.-H. Lee, and E.-S. Sim, "Analysis of delta-V losses during lunar capture sequence using finite thrust," Journal of Astronomy and Space Science, vol. 28, no. 3, pp. 203-216, 2011.

[16] S. Choi, Y. Song, J. Bae, E. Kim, and G. Ju, "Design and analysis of korean lunar orbiter mission using direct transfer trajectory," The Journal of the Korean Society for Aeronautical \& Space Sciences, vol. 41, no. 12, pp. 950-958, 2013.

[17] Y.-J. Song, S.-Y. Park, H.-D. Kim, and E.-S. Sim, "Development of precise lunar orbit propagator and lunar polar orbiter's 
lifetime analysis," Journal of Astronomy and Space Science, vol. 27, no. 2, pp. 97-106, 2010.

[18] D. H. Cho, B. Y. Jeong, D. H. Lee, and H. C. Bang, "Optimal perilune altitude of lunar landing trajectory," International Journal of Aeronautical and Space Sciences, vol. 10, no. 1, pp. 6774, 2009.

[19] B. Y. Jeong, Y. H. Choi, S. J. Jo, and H. C. Bang, "Terrain aided inertial navigation for precise planetary landing," Journal of the Korean Society for Aeronautical \& Space Sciences, vol. 38, no. 7, pp. 673-683, 2010.

[20] Y.-J. Song, S.-I. Ahn, S.-J. Choi, and E.-S. Sim, "Ground contact analysis for korea's fictitious lunar orbiter mission," Journal of Astronomy and Space Science, vol. 30, no. 4, pp. 255-267, 2013.

[21] Y. J. Song, S. J. Choi, S. I. Ahn, and E. S. Sim, "Analysis on tracking schedule and measurements characteristics for the spacecraft on the phase of lunar transfer and capture," Journal of Astronomy and Space Sciences, vol. 31, no. 1, pp. 51-61, 2014.

[22] S. Kim, D. Yoon, and K. Hyun, "Ground stations of korean deep space network for lunar explorations," The Journal of the Korean Society for Aeronautical \& Space Sciences, vol. 38, no. 5, pp. 499506, 2010.

[23] W. Lee, K. Cho, D. Yoon, and K. Hyun, "Design and performance analysis of downlink in space communications system for lunar exploration," Journal of Astronomy and Space Science, vol. 27, no. 1, pp. 11-20, 2010.

[24] W. Lee, D. Yoon, and J. Lee, "Performance analysis of maximum data rate for telemetry links in space communications for lunar explorations," Journal of the Korean Society for Aeronautical \& Space Sciences, vol. 39, no. 1, pp. 42-49, 2011.

[25] Y.-K. Kim, H.-D. Kim, J.-H. Lee, E.-S. Sim, and S.-W. Jeon, "Conceptual design of rover's mobility system for ground-based model," Journal of Astronomy and Space Sciences, vol. 26, no. 4, pp. 677-692, 2009.

[26] W.-S. Eom, Y.-K. Kim, J.-H. Lee, G.-H. Choi, and E.-S. Sim, "Study on a suspension of a planetary exploration rover to improve driving performance during overcoming obstacles," Journal of Astronomy and Space Science, vol. 29, no. 4, pp. 381387, 2012.

[27] T. J. Son, K. S. Na, J. H. Lim, K. W. Kim, and D. S. Hwang, "Development of a structure for lunar lander demonstrator," Aerospace Engineering and Technology, vol. 12, no. 1, pp. 213-220, 2013.

[28] W. B. Lee and D. Y. Rew, "Virtual flight test for conceptual lunar lander demonstrator," Aerospace Engineering and Technology, vol. 12, no. 1, pp. 87-93, 2013.

[29] S. S. Yang, Y. C. Kang, J. Y. Son, M. H. Oh, J. H. Kim, and J. Y. Cho, "Optimization of shock absorption system for lunar lander considering the effect of lunar regolith," The Journal of the Korean Society for Aeronautical \& Space Sciences, vol. 42, no. 4, pp. 284-290, 2014.

[30] K. J. Kim, J.-H. Lee, H. Seo et al., "An introduction to the lunar and planetary science activities in Korea," Advances in Space Research, vol. 54, no. 10, pp. 2000-2006, 2014.

[31] H. J. Lee, J. K. Lee, S.-M. Baek et al., "A CubeSat mission for Korean lunar exploration," in Proceedings of the 45th Lunar and Planetary Science Conference, p. 1783, Woodlands, Tex, USA, March 2014.

[32] I. Garrick-Bethell, R. P. Lin, H. Sanchezd et al., "Lunar magnetic field measurements with a cubesat," in Sensors and Systems for Space Applications VI, vol. 8739 of Proceedings of SPIE, Baltimore, Md, USA, April 2013.
[33] T. Komarek, Z. Bailey, H. Schone, T. Jedrey, and A. Chandler, "Novel ideas for exploring Mars with CubeSats: challenges and possibilities," in Proceedings of the 10th IAA Low-Cost Planetary Missions Conference, Pasadena, Calif, USA, June 2013.

[34] NASA Jet Propulsion Laboratory, "JPL Selects Europa CubeSat Proposals for Study," October 2014, http://www.jpl.nasa.gov/ news/news.php? feature $=4330$.

[35] H. Jin, J. Seon, K. H. Kim et al., "The system design of TRIO cinema mission," in Proceedings of the 38th COSPAR Scientific Assembly, p. 12, Bremen, Germany, July 2010.

[36] H. Jin, I. Garrick-Bethell, Y. J. Song et al., "Preliminary analysis of a lunar CubeSat impactor mission," in Proceedings of the Lunar Science Workshop, p. 13, Yongin, Republic of Korea, May 2014.

[37] D. A. Vallado, Fundamentals of Astrodynamics and Applications, Kluwer Academic Publishers, 3rd edition, 2007.

[38] H. D. Curtis, Orbital Mechanics for Engineering Students, Elsevier Aerospace Engineering Series, Butterworth-Heinemann, 2nd edition, 2009.

[39] R. P. Brent, Algorithms for Minimization without Derivatives, Dover, 2002.

[40] E. M. Sodano, "General non-iterative solution of the inverse and direct geodetic problems," Bulletin Géodésique, vol. 39, no. 1, pp. 69-89, 1965.

[41] E. M. Standish, "JPL planetary and lunar ephemerides, DE405/ LE405," Jet Propulsion Laboratory Interoffice Memorandum IOM 312.F-98-048, 1998.

[42] W. Lan, R. Munakata, L. R. Nugent, and D. Pignatelli, "Poly picosatellite orbital deployer Mk. III Rev. E user guide," The CubeSat Program CP-PPODUG-1.0-1, California Polytechnic State University, 2007.

[43] L. L. Hood and G. Schubert, "Lunar magnetic anomalies and surface optical properties," Science, vol. 208, no. 4439, pp. 49$51,1980$.

[44] D. Hemingway and I. Garrick-Bethell, "Magnetic field direction and lunar swirl morphology: insights from Airy and Reiner Gamma," Journal of Geophysical Research: Planets, vol. 117, no. 10, Article ID E10012, 2012. 


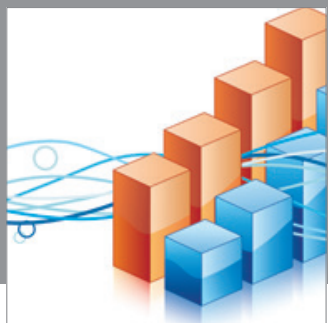

Advances in

Operations Research

mansans

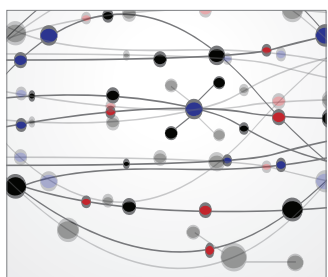

The Scientific World Journal
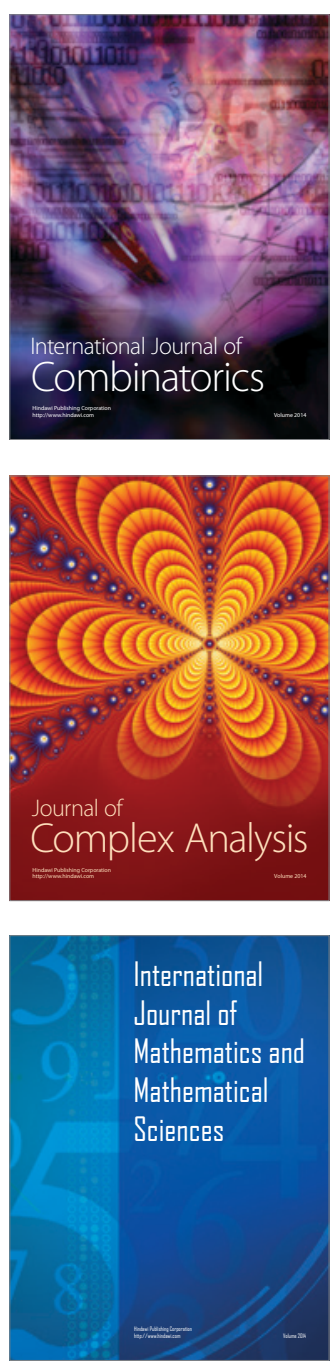
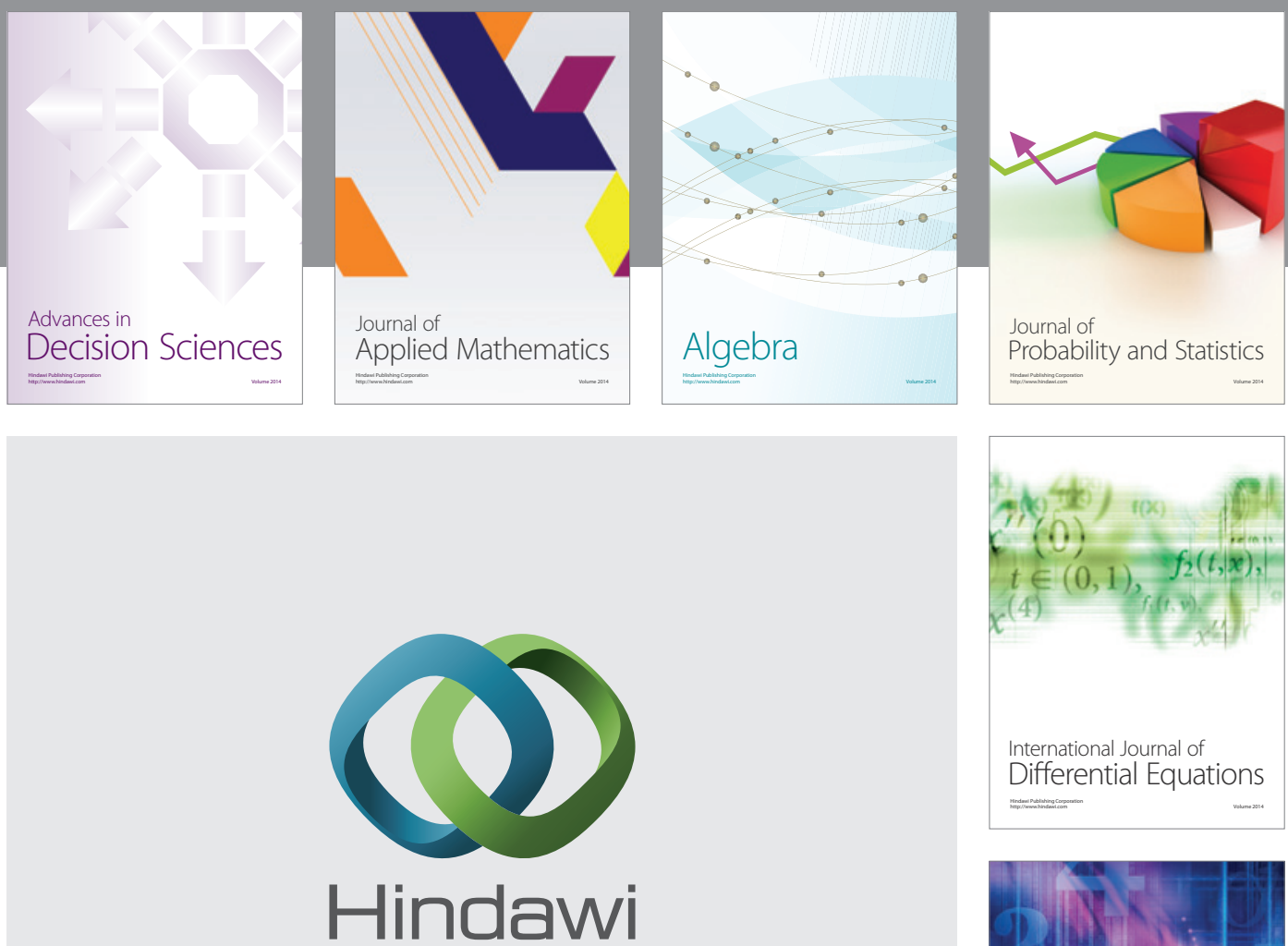

Submit your manuscripts at http://www.hindawi.com
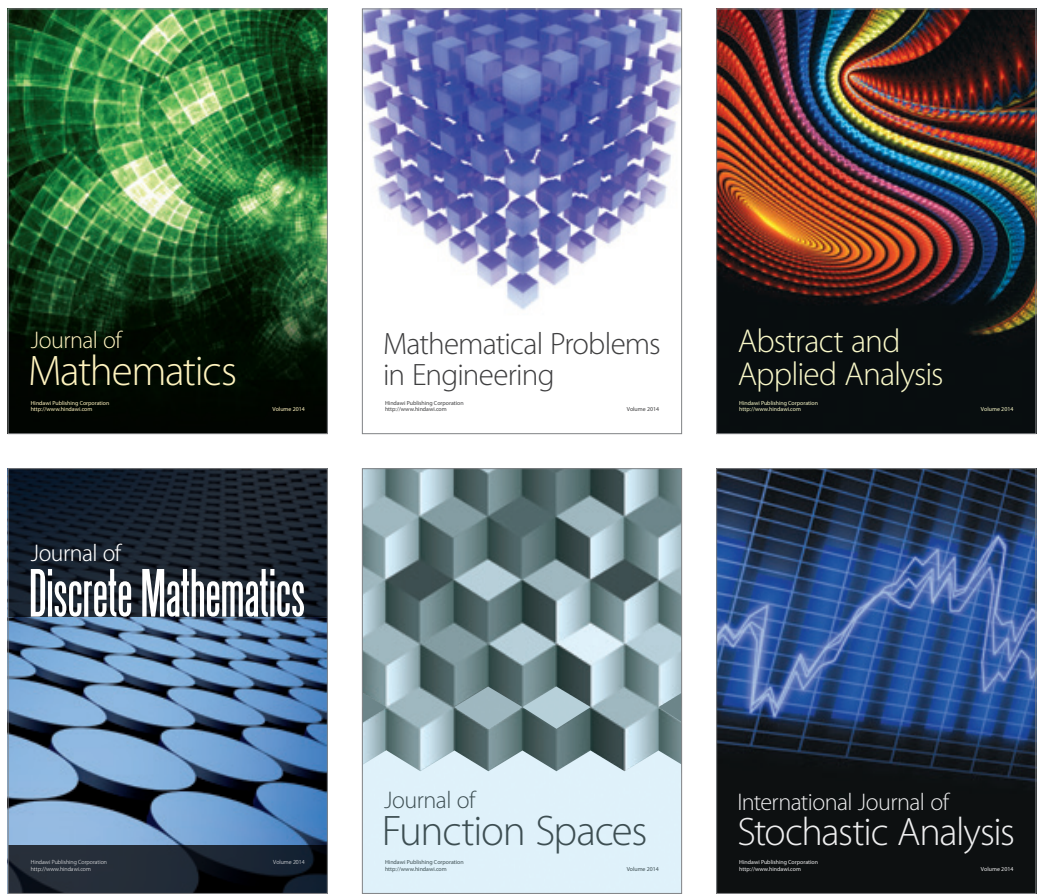

Journal of

Function Spaces

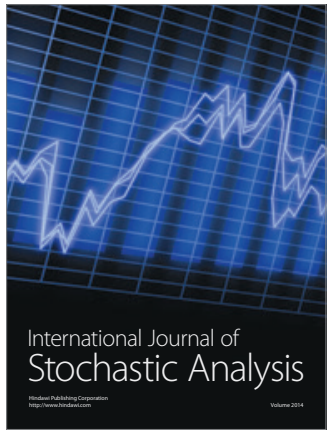

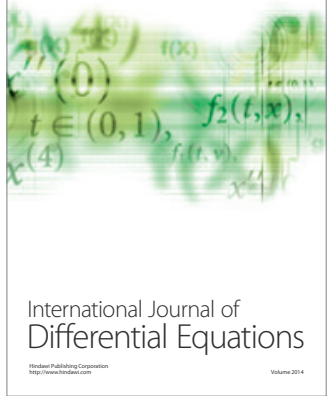
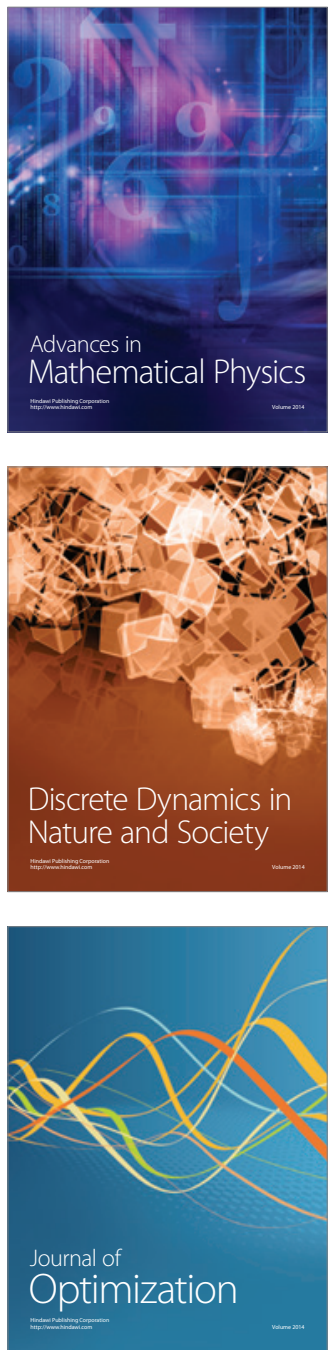